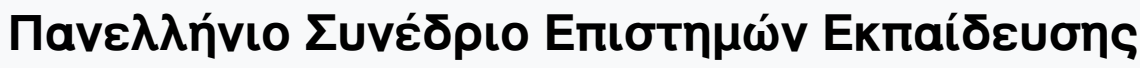

Tóp. 2016, Ap. 2

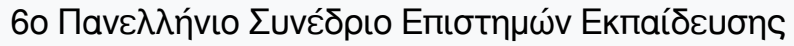

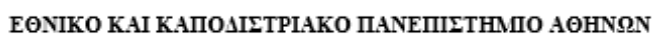
TOMEA EISIKHE AГתГHE KAI YYXOAOГIAE П.T.A.E.

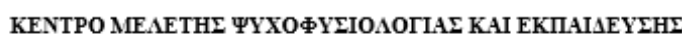

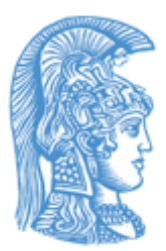

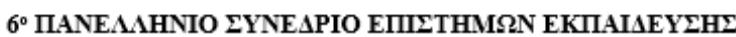

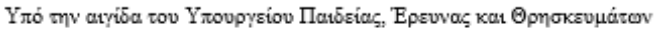

\section{Dyslexia: a different way of learning}

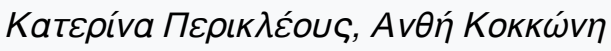

doi: $\underline{10.12681 / \text { edusc. } 1000}$

\section{ПIPAKTIKA IYNEAPIOY}

ISSN: 2529-1157

\section{EIIMEAEIA:}

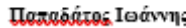

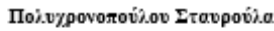

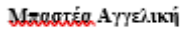

IOYNIOE 2016

\section{Bıß入ıорачıкń avaчopá:}

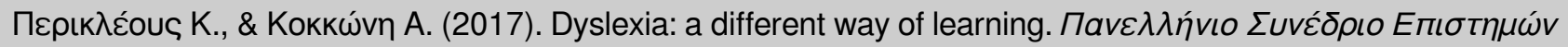

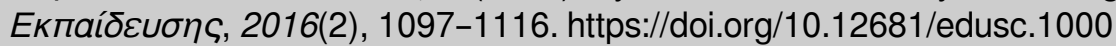




\title{
Dyslexia: a different way of learning
}

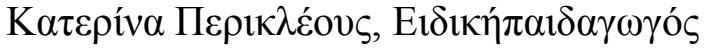 \\ perikatie@hotmail.com

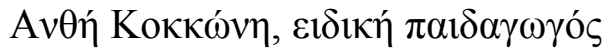 \\ info@leximathia.gr
}

\section{Пєрі́ $\eta \psi \eta$}

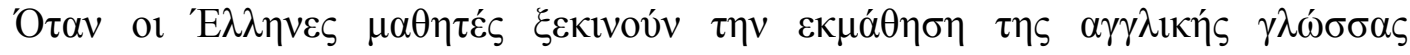

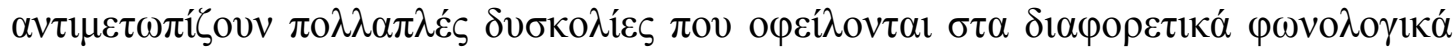

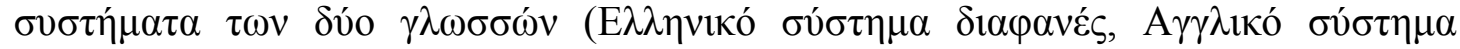

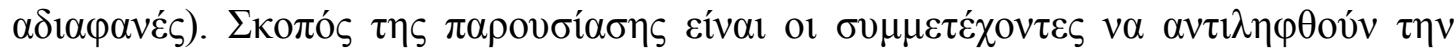

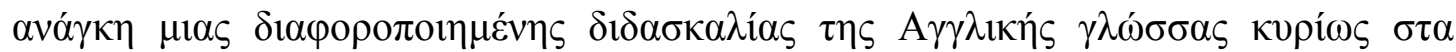

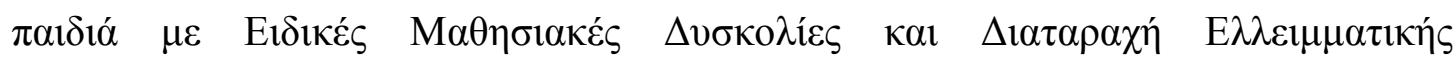

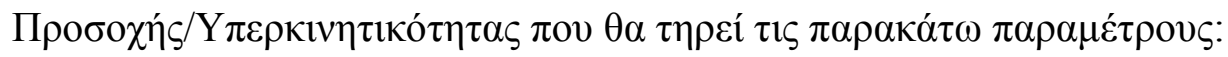

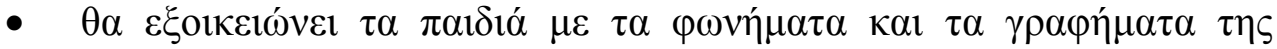
A $\gamma \gamma \lambda \iota \kappa n ́ \varsigma ~ \gamma \lambda \omega ́ \sigma \sigma \alpha \varsigma$

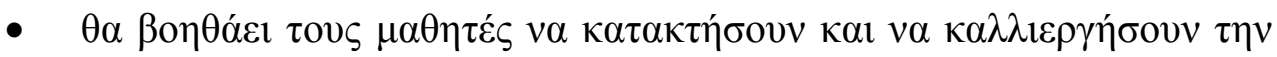

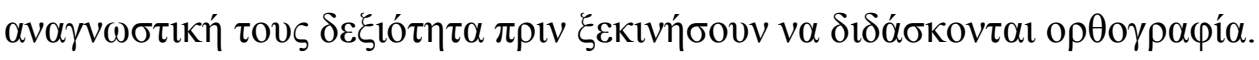

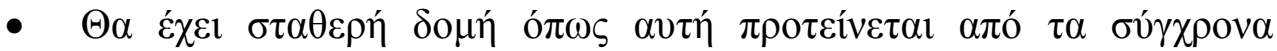

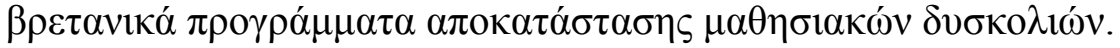

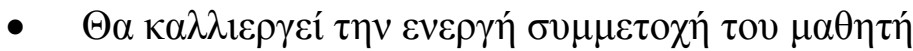

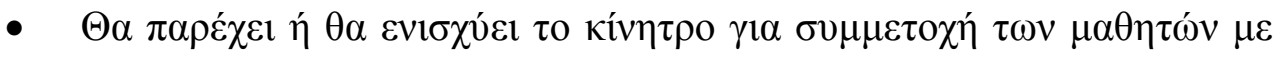

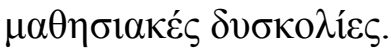

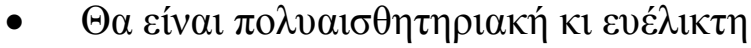

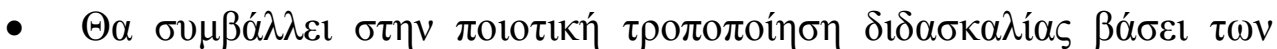

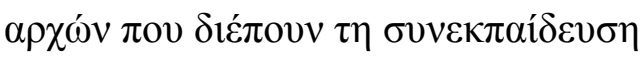

\section{$\Lambda \varepsilon ́ \xi \varepsilon เ \varsigma-\kappa \lambda \varepsilon เ \delta ı \alpha ́$}




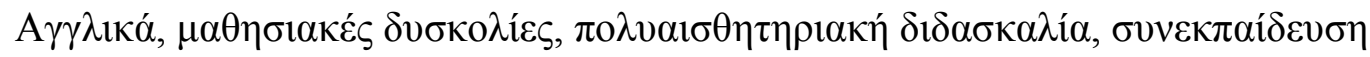

\begin{abstract}
It has been reported that when Greek pupils start learning English many difficulties emerge, mainly due to the different phonological systems of the two languages. In Greek there is a phonemic correspondence between the phonemes and the graphemes whereas this correspondence does not always apply to English. The present paper aims at explaining the need to differentiate the way the English language is taught so as to respond to the needs of all learners, especially of those pupils with learning difficulties. In order for this differentiation to be put in practice, the following aspects should be taken into consideration:
\end{abstract}

- Greek pupils need time so as to practice the English phonemes and graphemes.

- Greek pupils need to learn to read before they start writing.

- Teaching should be structured and cover the pupils' educational needs.

- Pupils should be inspired to participate in the learning process.

- Pupils with learning difficulties should feel included and motivated.

- $\quad$ The teaching approach should be multimodal and flexible.

\title{
Key words
}

English, learning difficulties, multisensory approach, inclusion

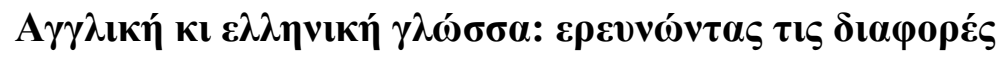

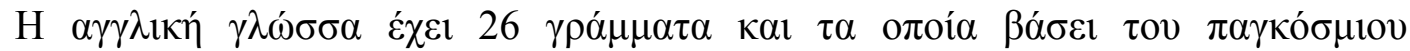

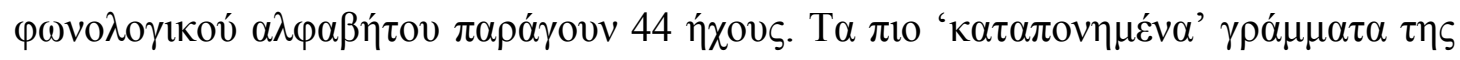

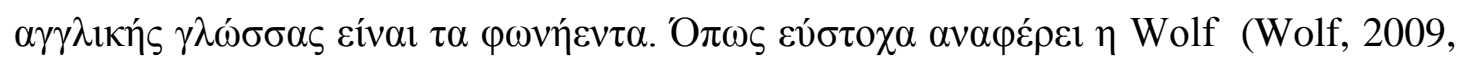

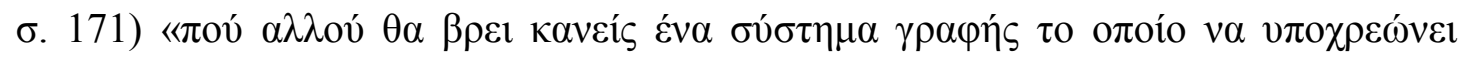




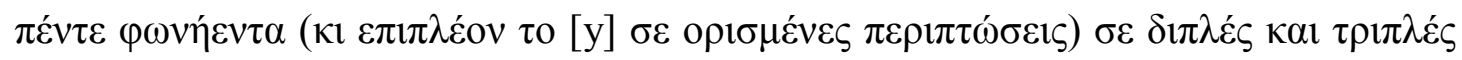

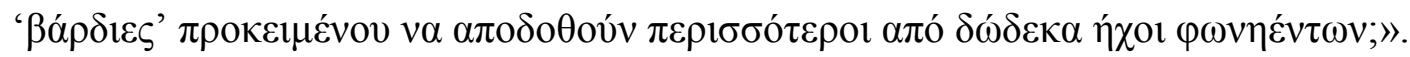

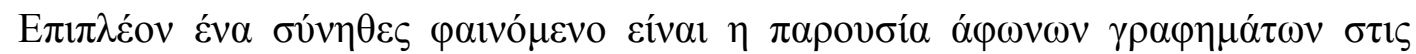

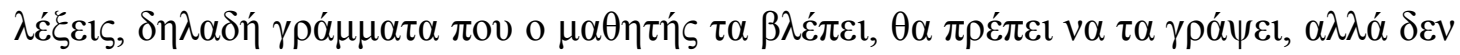

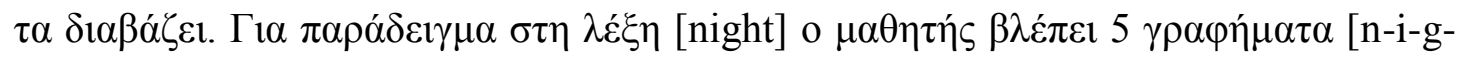

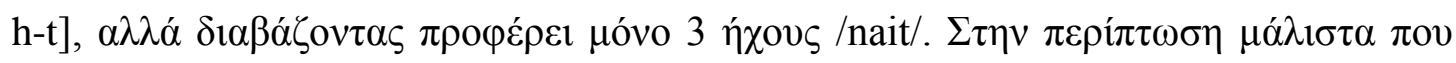

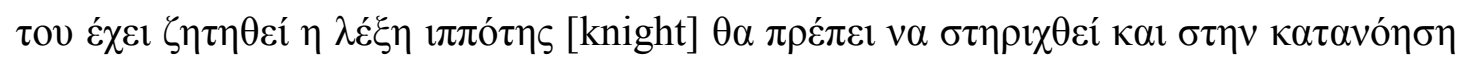

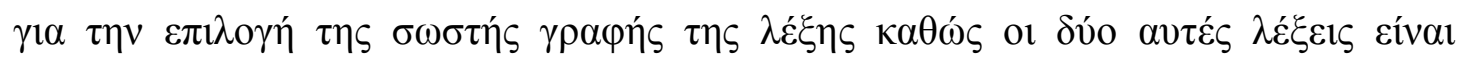

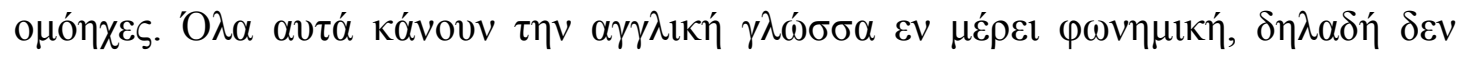

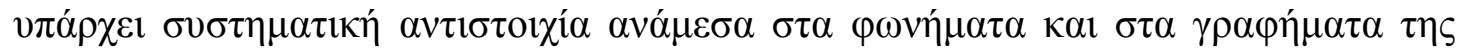

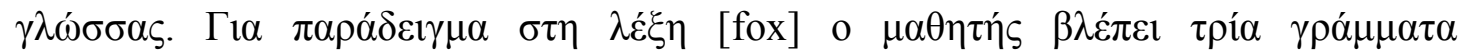

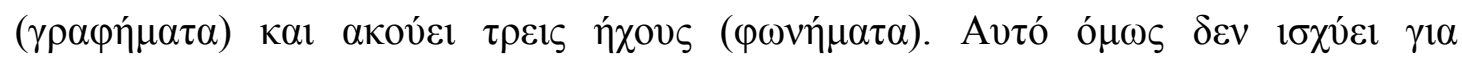

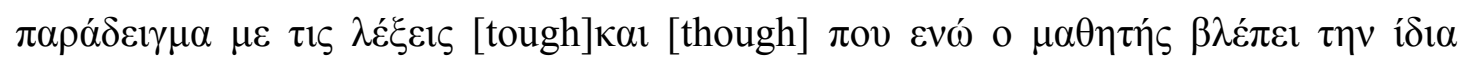

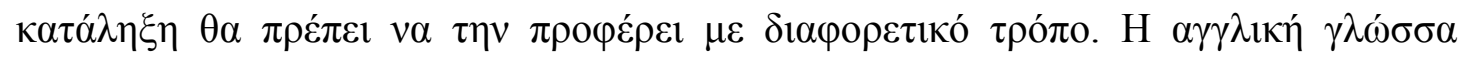

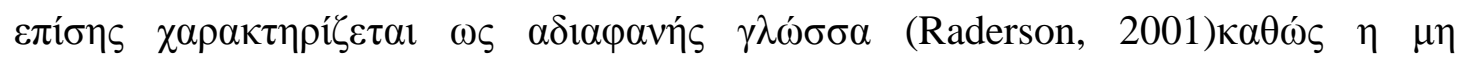

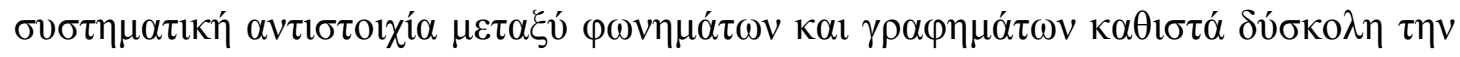

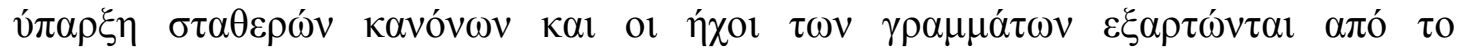

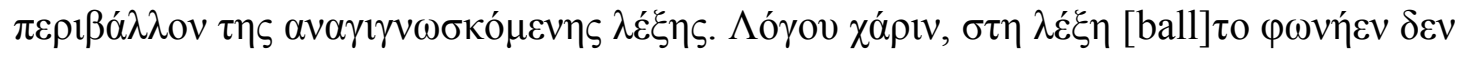

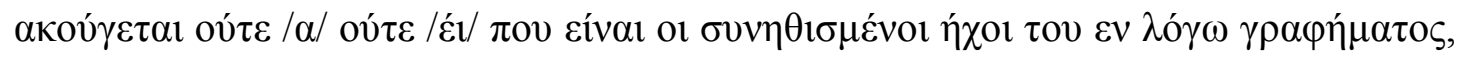
$\alpha \lambda \lambda \alpha \dot{\alpha} / \mathrm{o} /$.

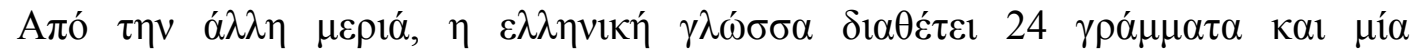

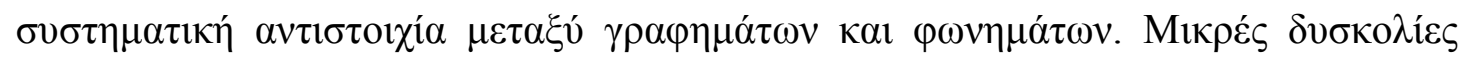

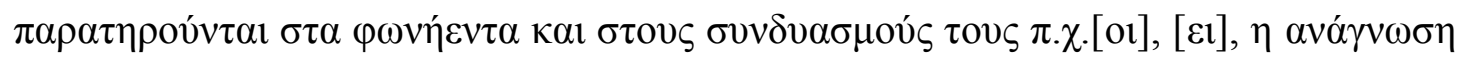

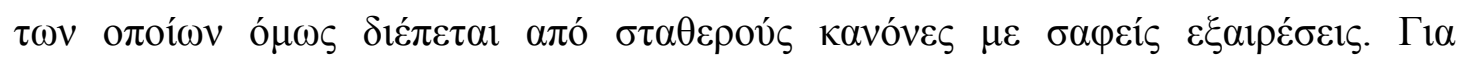

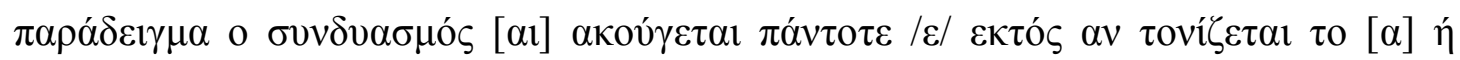

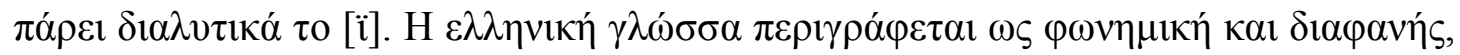

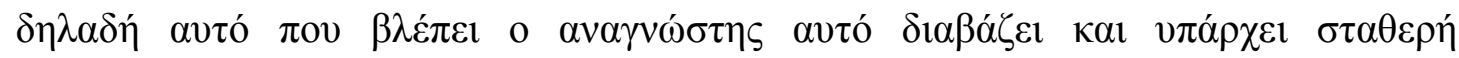

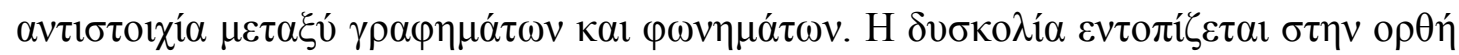

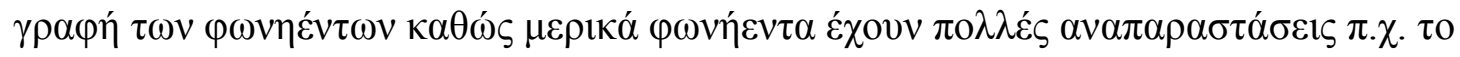

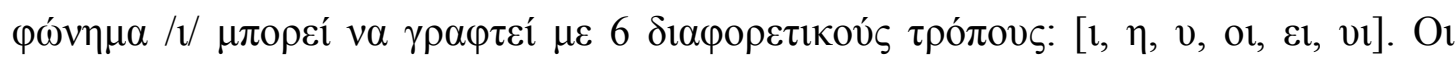

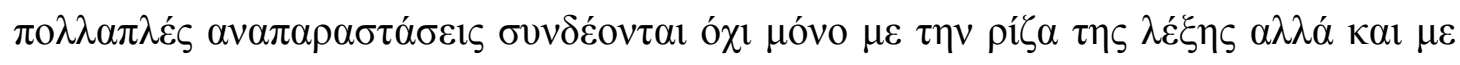

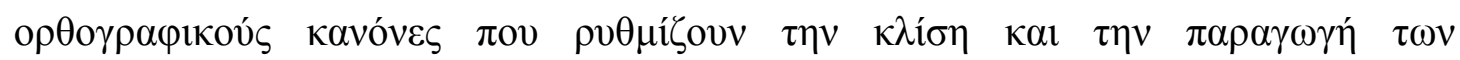
$\lambda \varepsilon \dot{\varepsilon} \xi \omega v .(\mathrm{A} \gamma \gamma \varepsilon \dot{\lambda} \lambda \mathrm{ov}, 2007)$ 


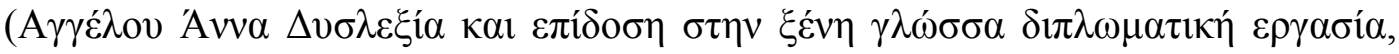

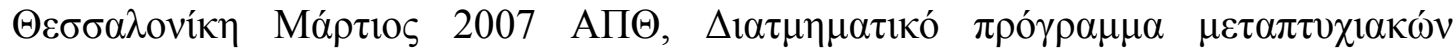

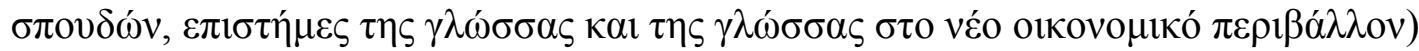

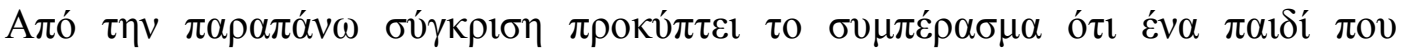

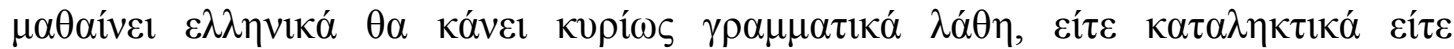

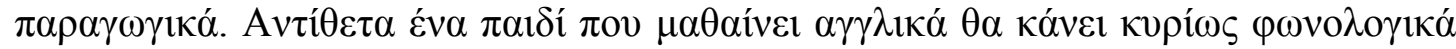

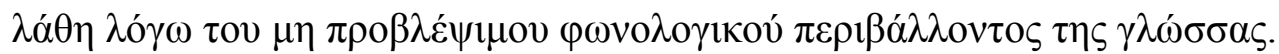

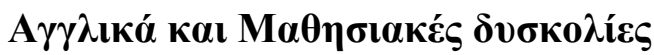

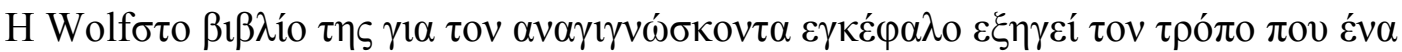

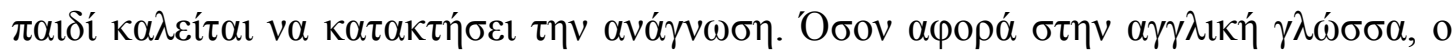

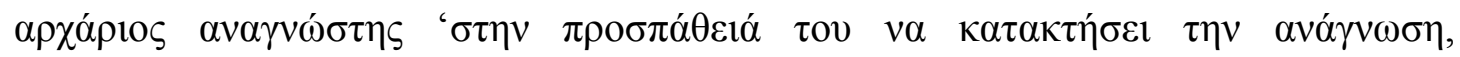

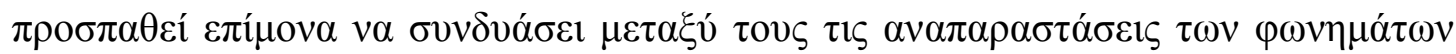

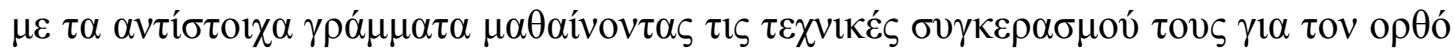

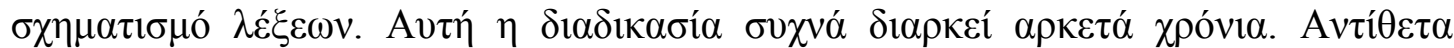

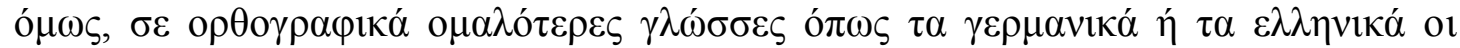

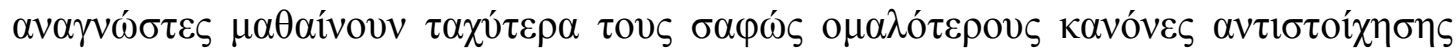

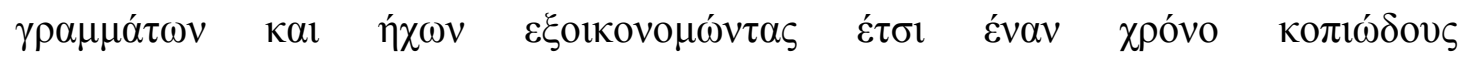

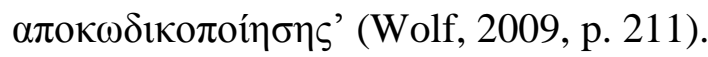

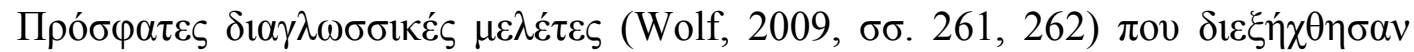

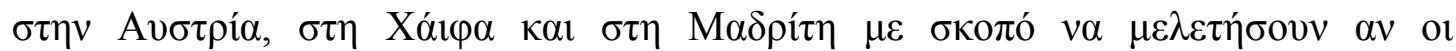

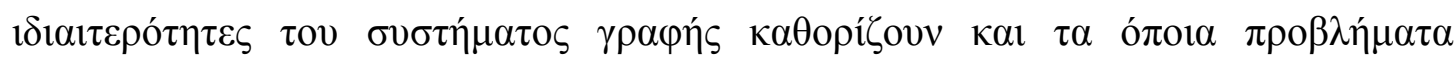

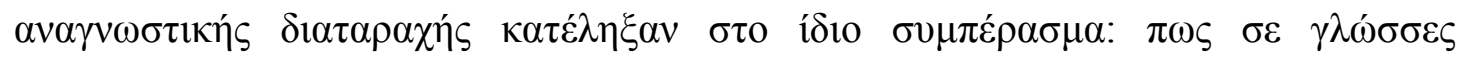

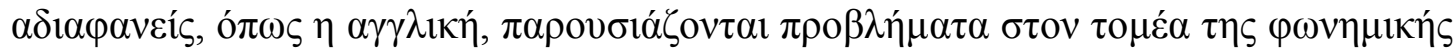

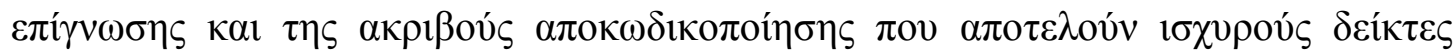

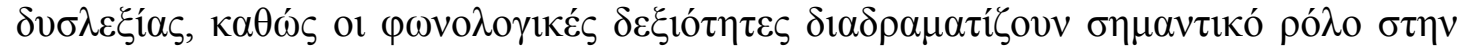

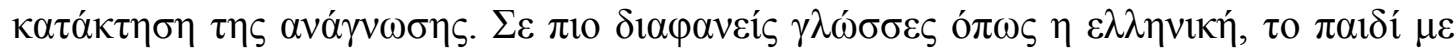

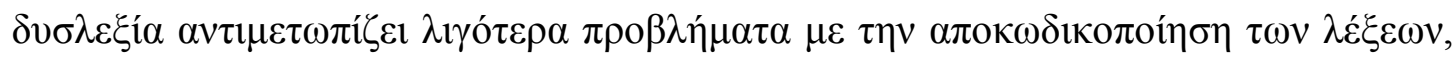

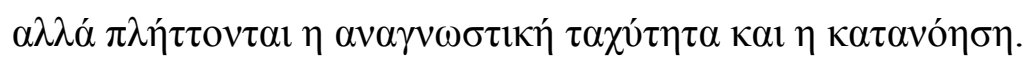




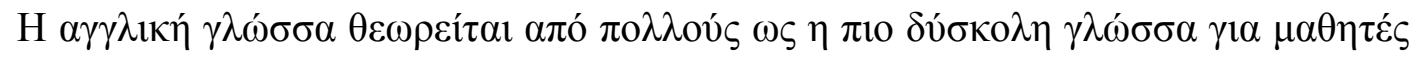

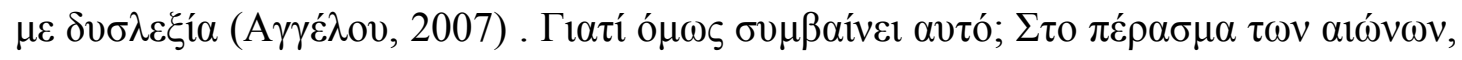

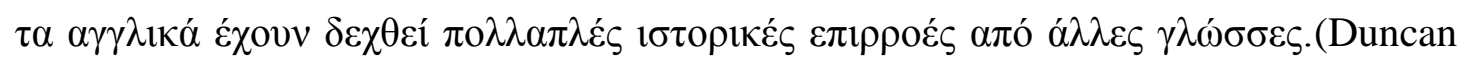

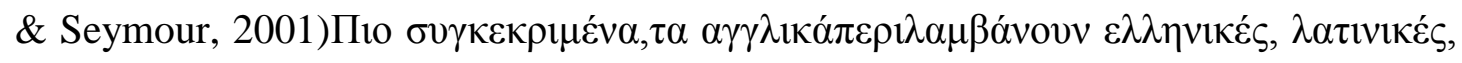

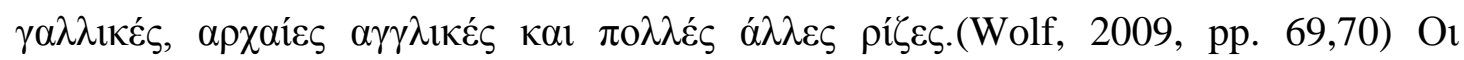

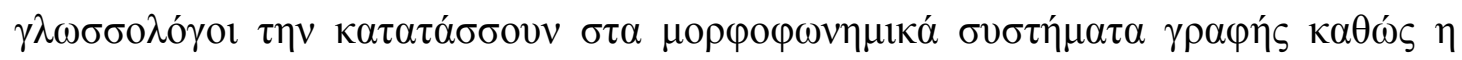

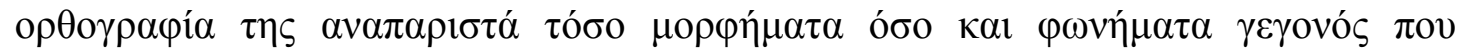

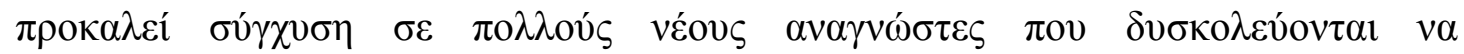

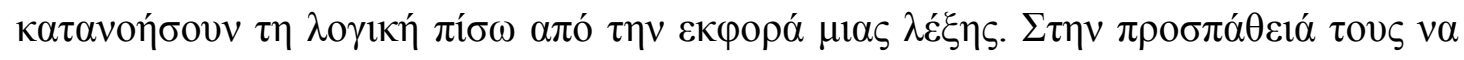

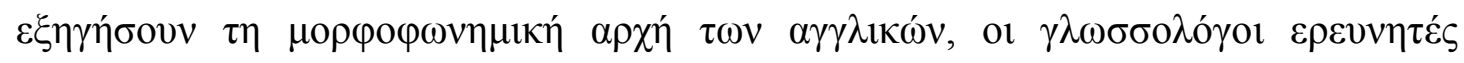

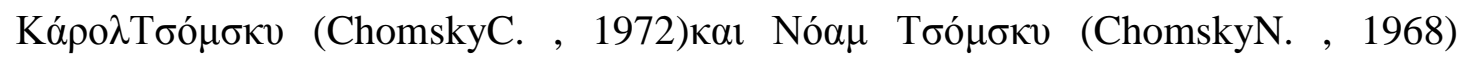

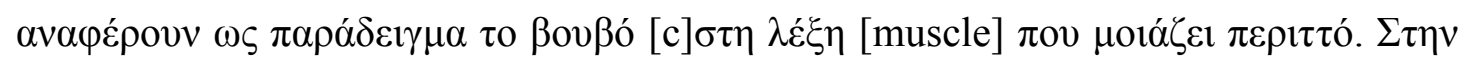

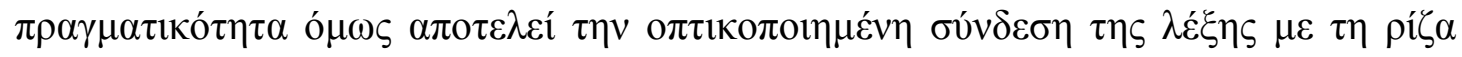

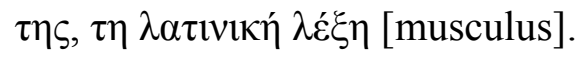

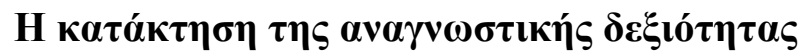

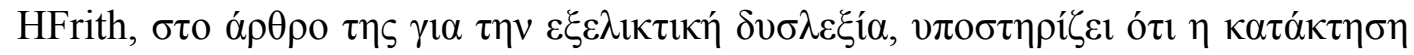

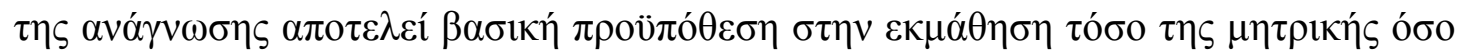

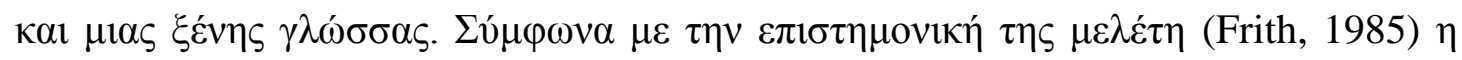

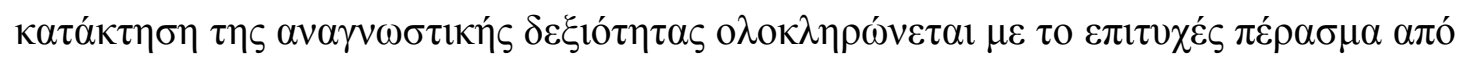
$\tau \alpha \varepsilon \xi \eta \dot{\eta} \varsigma \tau \rho i ́ \alpha \sigma \tau \alpha \dot{\delta} 1 \alpha$ :

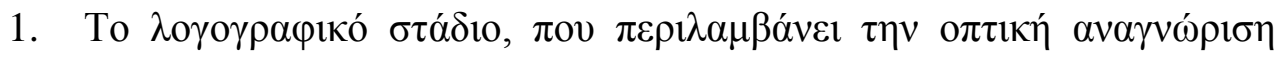

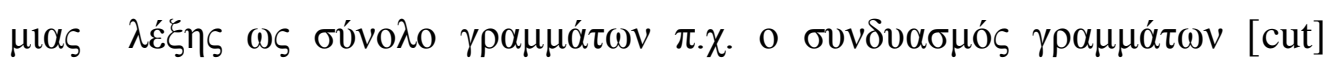

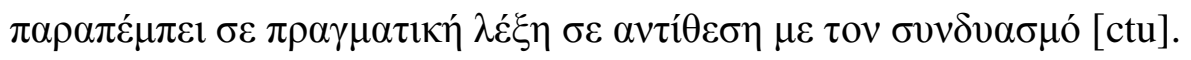

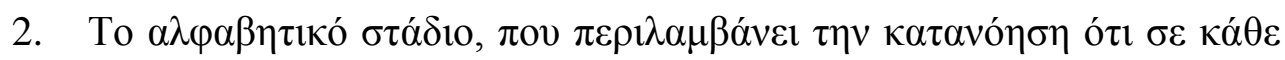

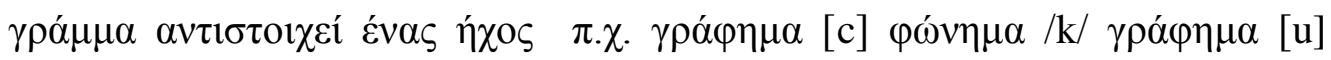
$\varphi \omega ́ v \eta \mu \alpha / a ́ / ~ \gamma \rho \alpha ́ \varphi \eta \mu \alpha[t] \varphi \omega ́ v \eta \mu \alpha / t /$

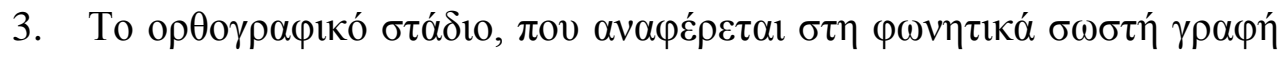

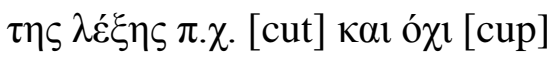




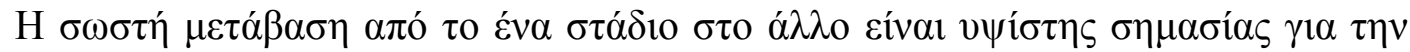

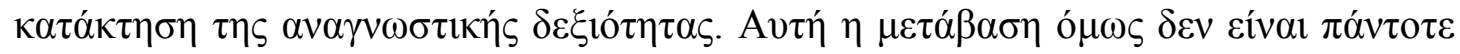

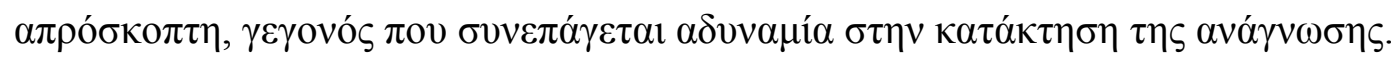

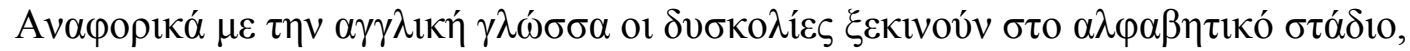

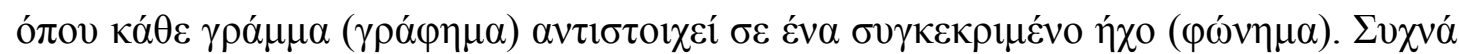

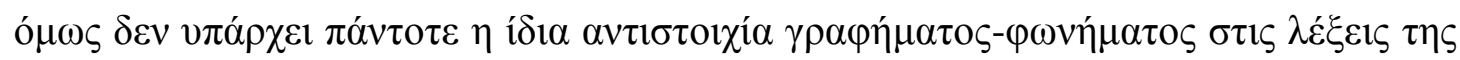

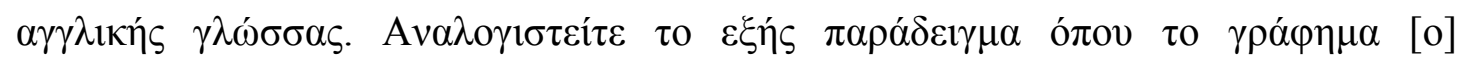

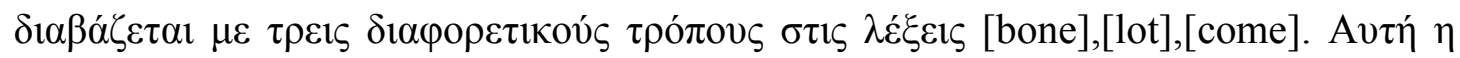

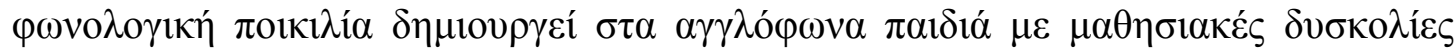

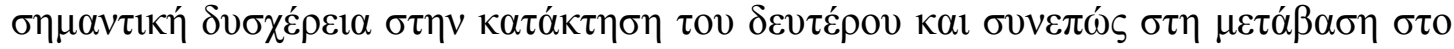

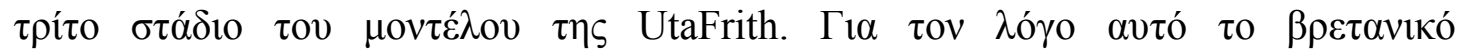

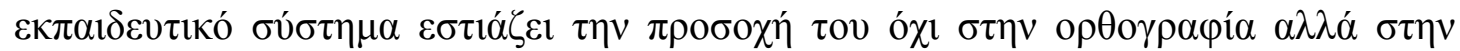

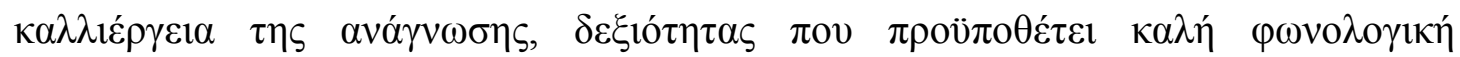

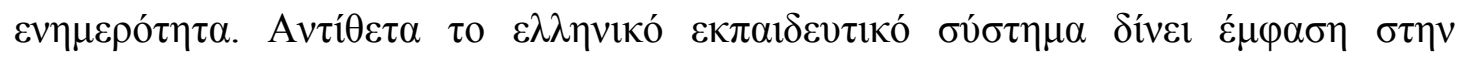

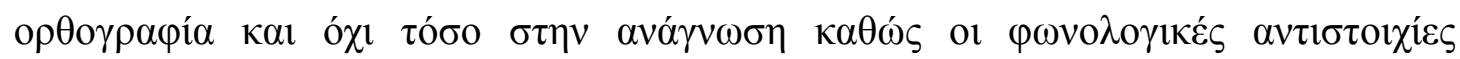

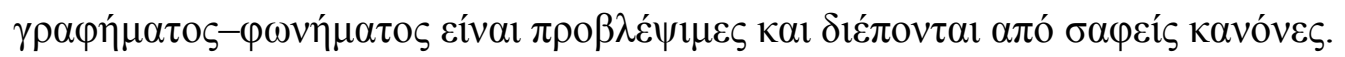

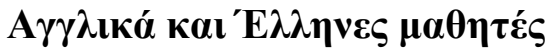

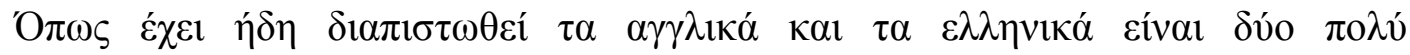

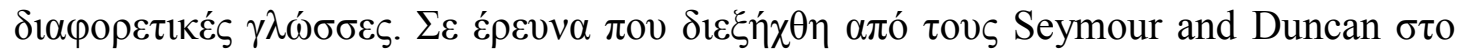

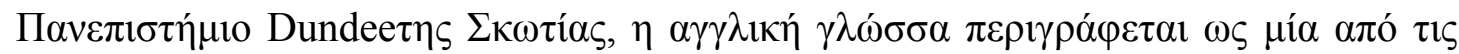

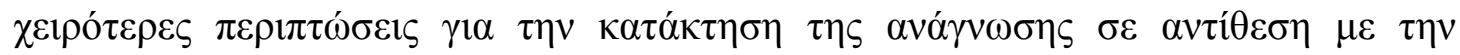

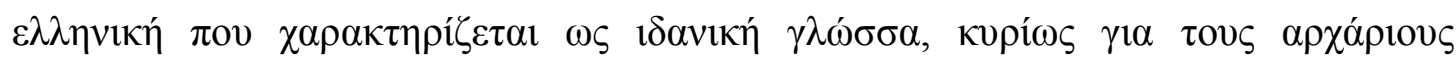

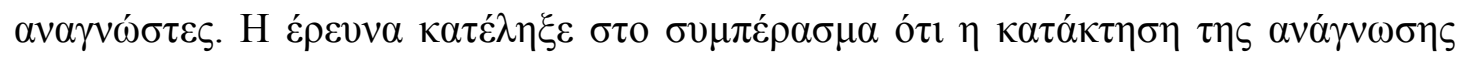

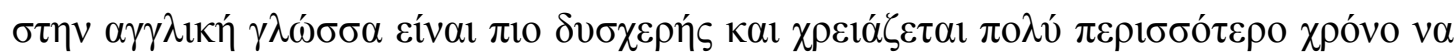

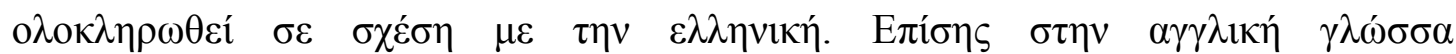

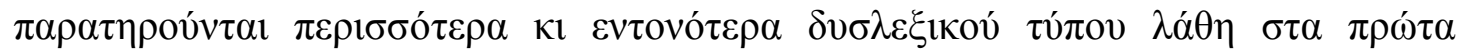
$\sigma \tau \alpha \dot{\delta} \delta \alpha \kappa \alpha \tau \alpha ́ \kappa \tau \eta \sigma \eta \varsigma \tau \eta \varsigma \alpha \nu \alpha \gamma \nu \omega \sigma \tau \iota \kappa \eta ́ \varsigma ~ \delta \varepsilon \xi 1 o ́ \tau \eta \tau \alpha \varsigma .(D u n c a n \& S e y m o u r, 2001)$

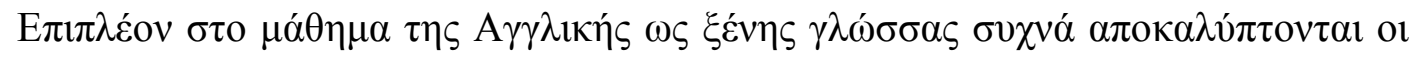

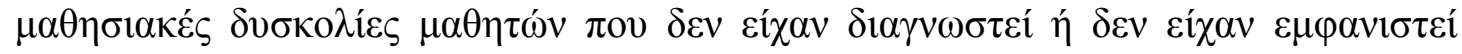

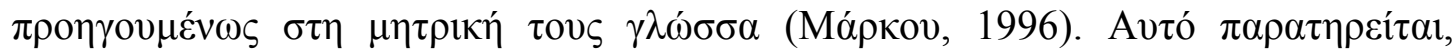




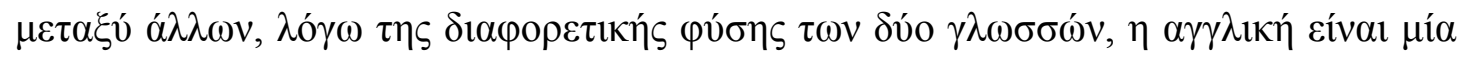

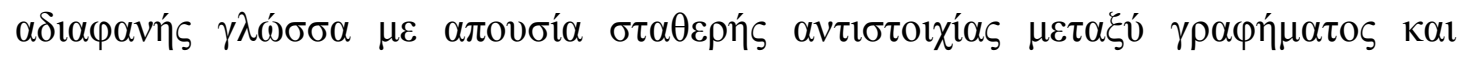

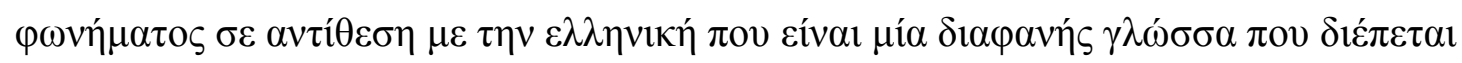

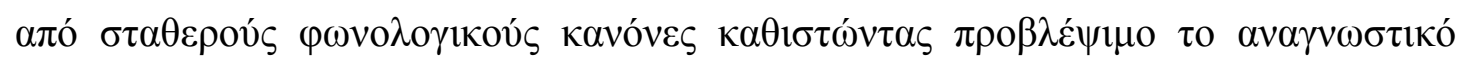
$\pi \varepsilon \rho 1 \beta a ́ \lambda \lambda$ ov.

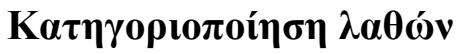

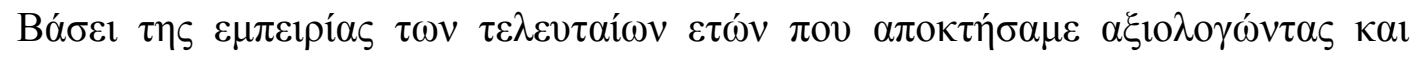

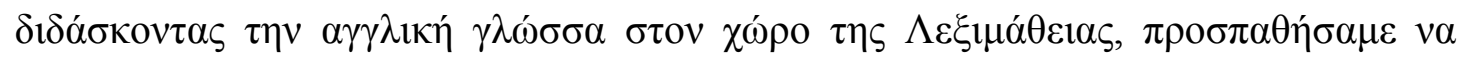

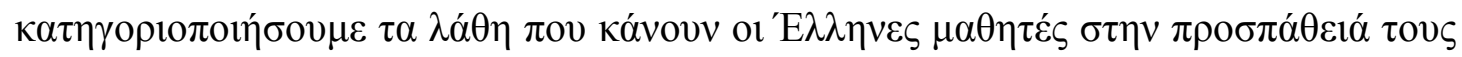

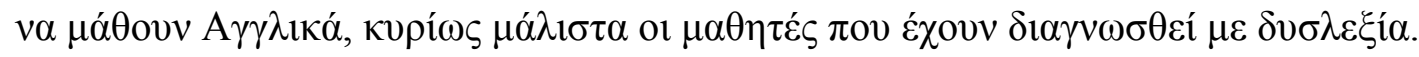

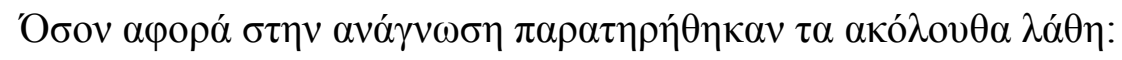

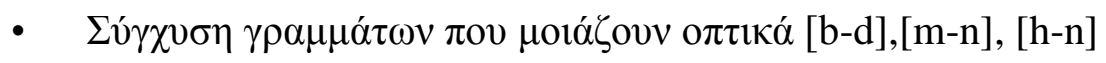

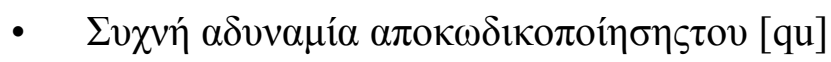

- $\Delta v \sigma \kappa o \lambda i ́ \alpha \sigma \tau \eta v \alpha v \alpha ́ \gamma v \omega \sigma \eta \varphi \omega v \eta \varepsilon v \tau \iota \kappa \omega ́ v \sigma v v \delta v \alpha \sigma \mu \omega ́ v \pi . \chi$. /haus//koat/

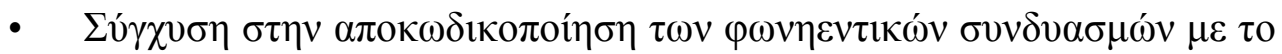

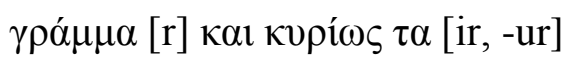

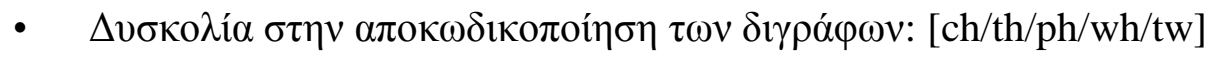

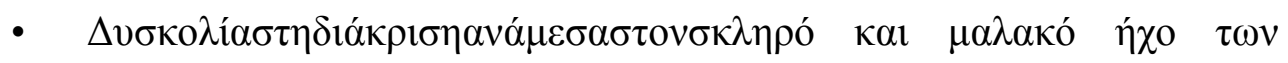
$\gamma \rho \alpha \mu \mu \alpha ́ \tau \omega \nu$ [c] $\kappa \alpha \mathrm{l}$ [g]e.g[leg]vs. [gel][cat]vs[pencil].

- $\quad \Sigma u ́ \gamma \chi v \sigma \eta \mu \varepsilon \tau \eta v \varepsilon \kappa \varphi о \rho \alpha ́ \tau \eta \varsigma \kappa \alpha \tau \alpha ́ \lambda \eta \xi \eta \varsigma e d \sigma \tau o v \operatorname{simplepast\kappa \alpha \iota \sigma \tau \eta vpastparti~}$

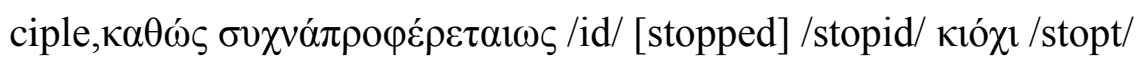

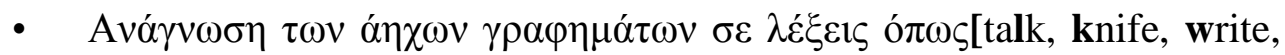
island]

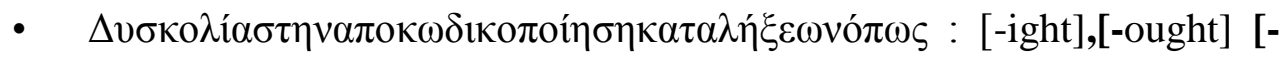

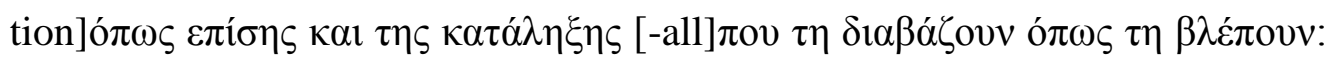

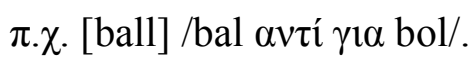

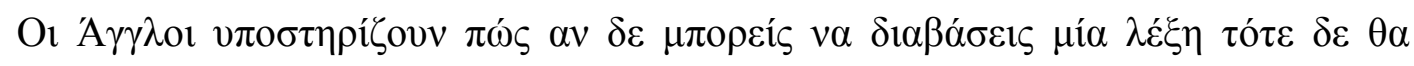

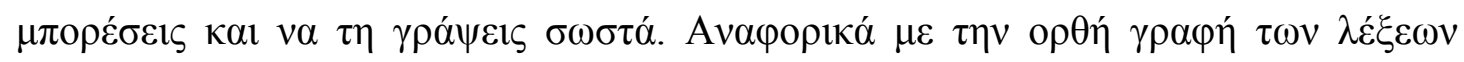

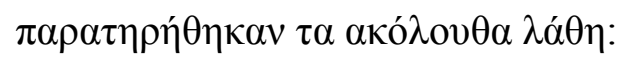




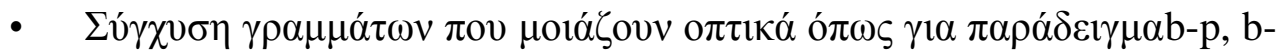
d

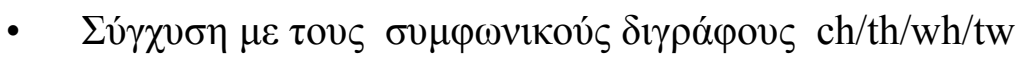

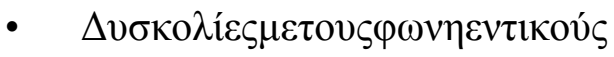

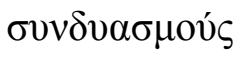

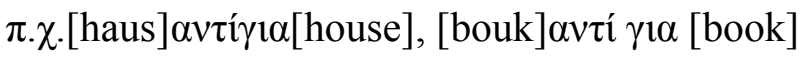

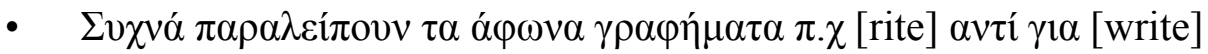

- $\quad \Sigma 0 ́ \gamma \chi v \sigma \eta \mu \varepsilon \tau \iota \varsigma \kappa \alpha \tau \alpha \lambda \eta ́ \xi \varepsilon 1 \varsigma$-: [-ight],[-ought] [-tion]

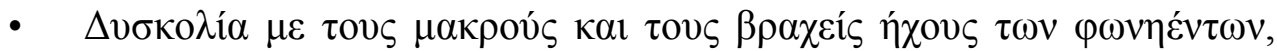

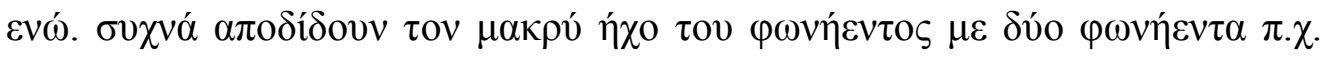
[meik] $\alpha v \tau^{\prime} \gamma 1 \alpha$ [make]

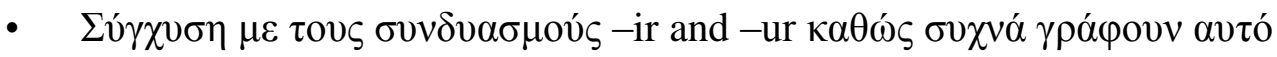

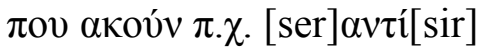

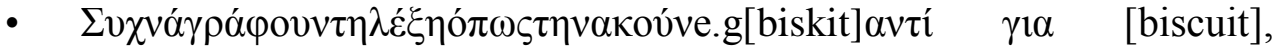

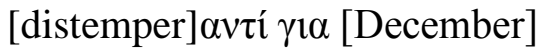

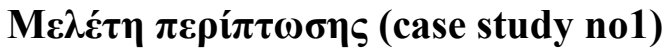

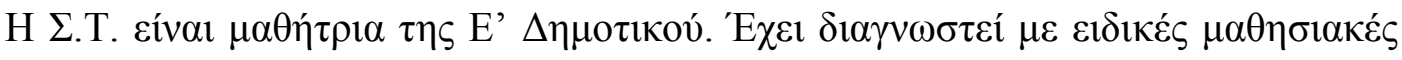

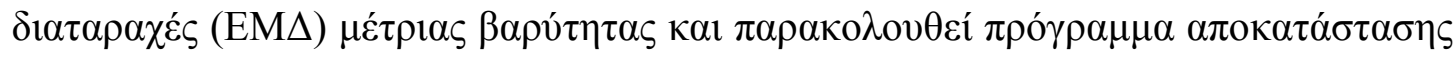

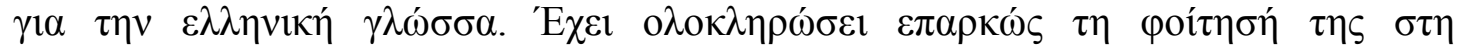

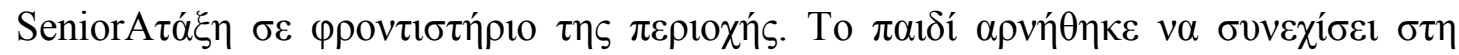

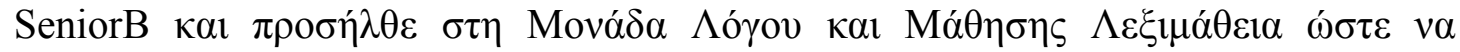

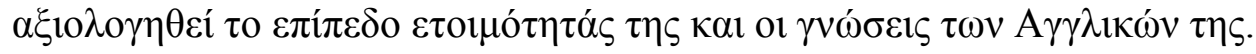

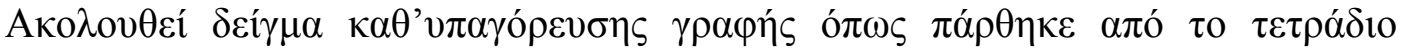

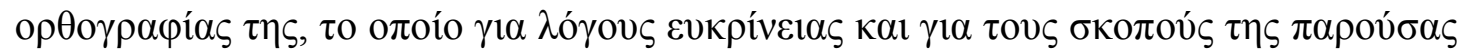
$\pi \alpha \rho 0 v \sigma i ́ \alpha \sigma \eta \varsigma \varepsilon ́ \chi \varepsilon ı 1 \delta \alpha \chi \tau v \lambda o \gamma \rho \alpha \varphi \eta \theta \varepsilon i ́(\varepsilon ı \kappa o ́ v \alpha 1)$ 


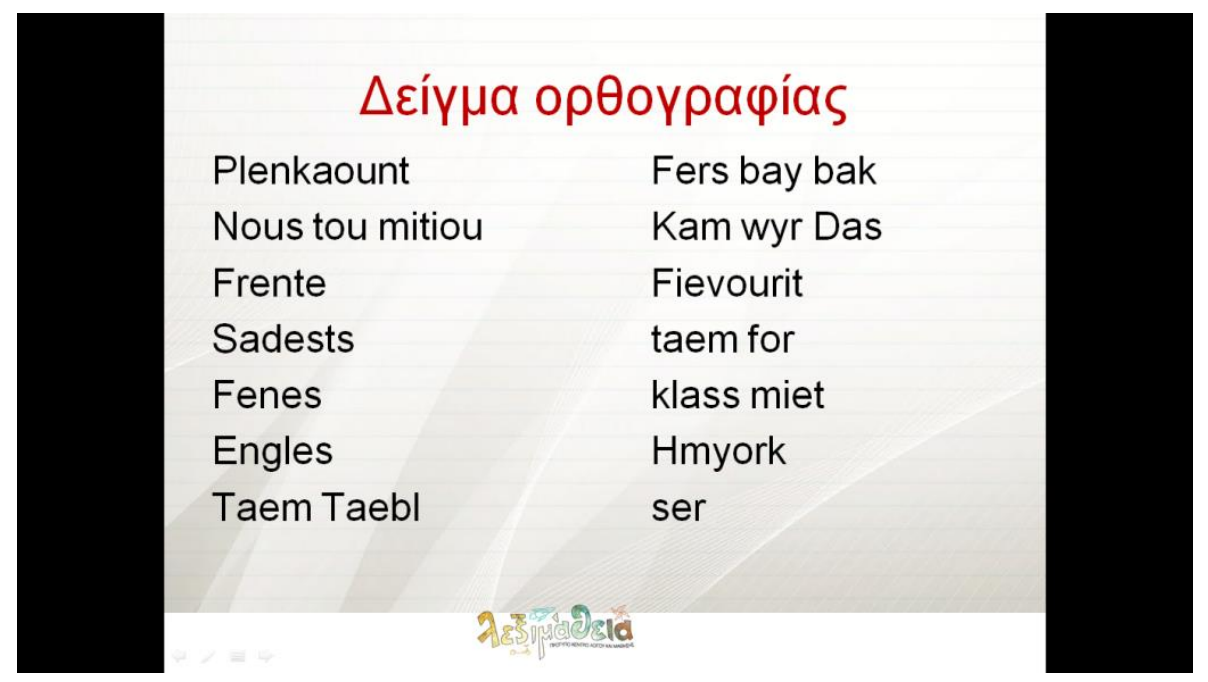

Eıкóva 1

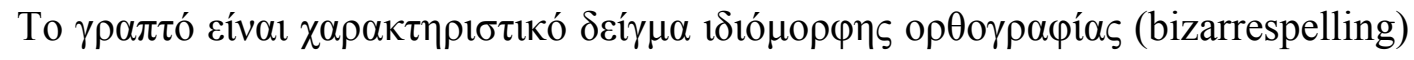

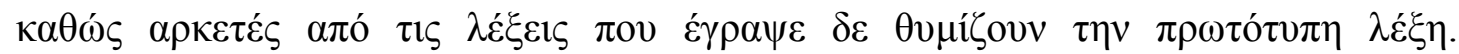

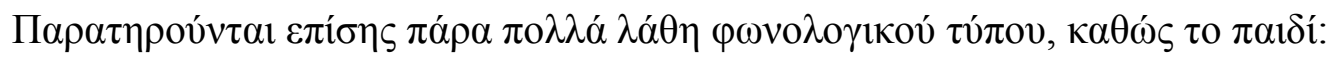

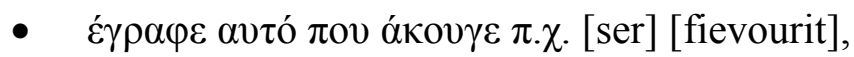

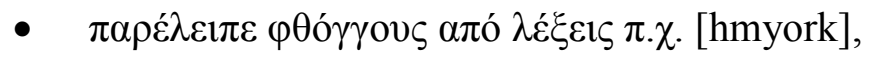

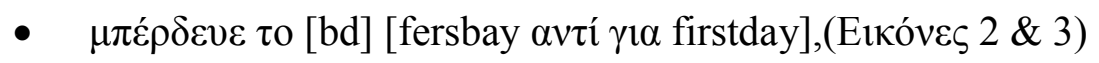

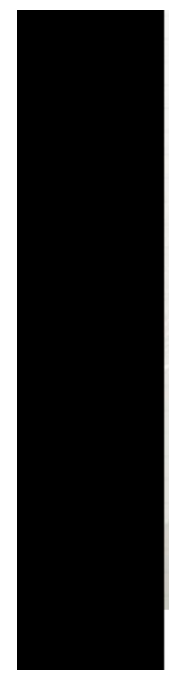

Акоибะ ดอ

- playground

- nice to meet you

- French

- subjects

- finish

- English

- time table
'Eүрачe plenkaount nous tou mitiou Frente sadests

fenes

Engles

taem taebl

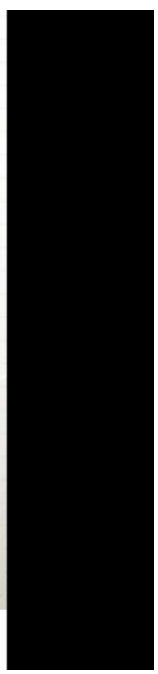


E1кóva 2

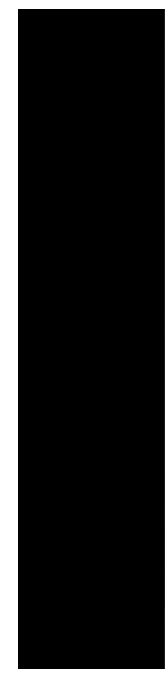

Акоибє

- sir

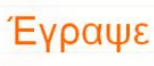

ser

- first day back

fers bay bak

- come with us kam wyr Das

- favourite fievourit

- time for taem for

- classmate klass miet

- homework Hmyork

Eıкóva 3

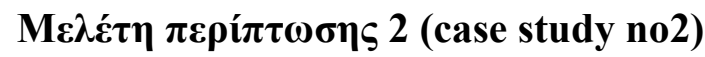

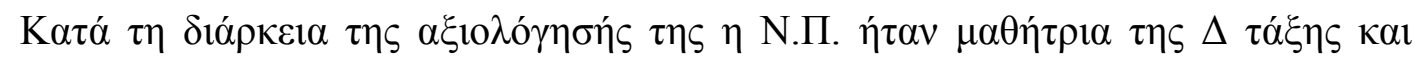

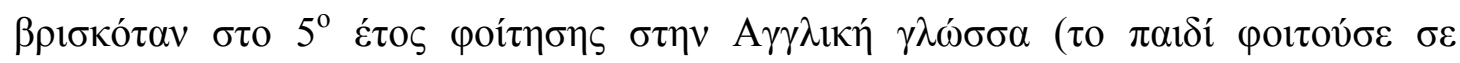

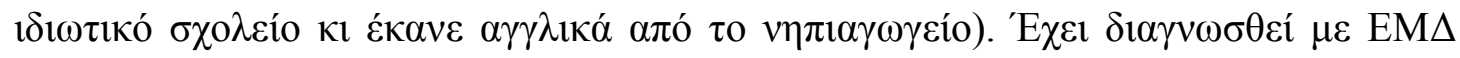

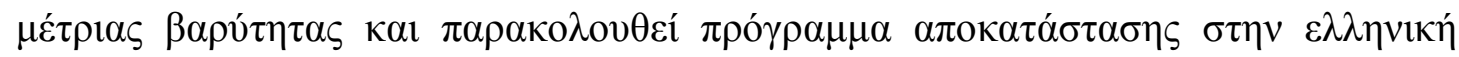
$\gamma \lambda \omega ́ \sigma \sigma \alpha$.

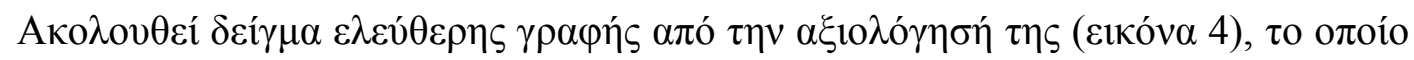

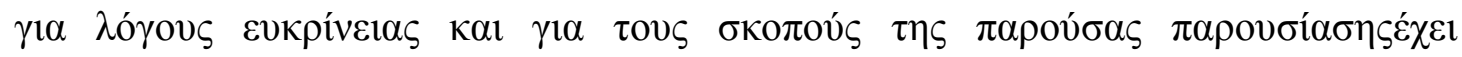
$\delta \alpha \chi \tau v \lambda о \gamma \rho \alpha \varphi \eta \theta \varepsilon i ́ . ~(\varepsilon 1 \kappa o ́ v \alpha 5)$ 


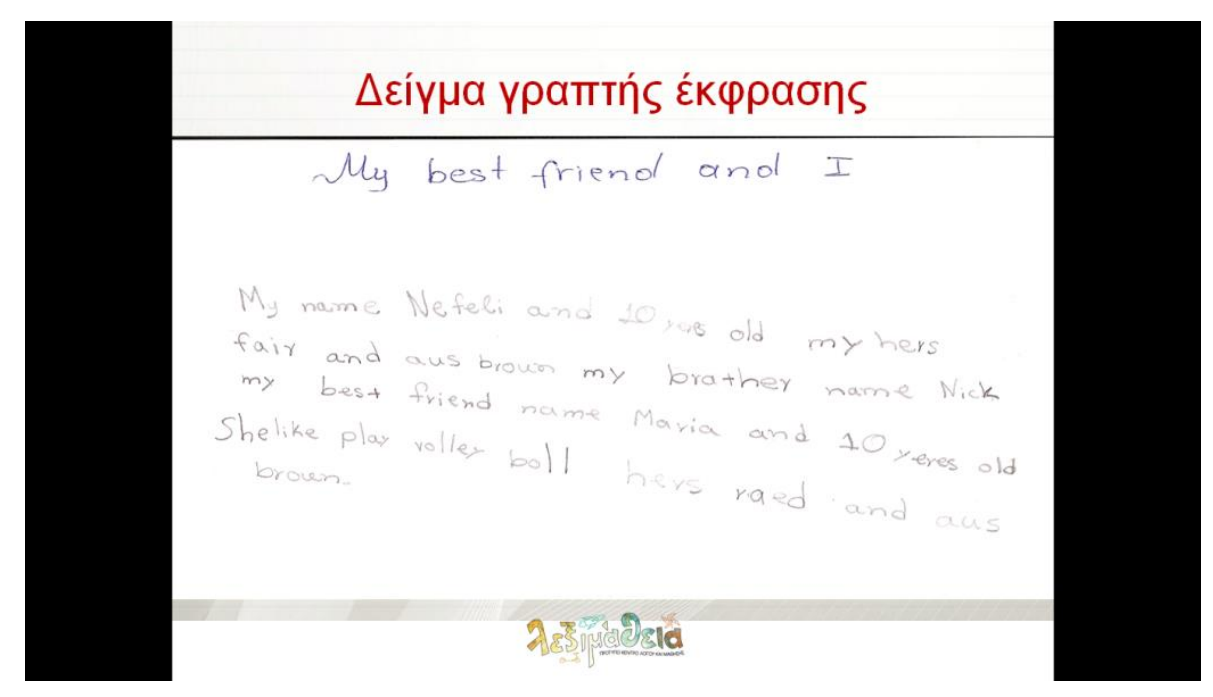

Eıкóva 4

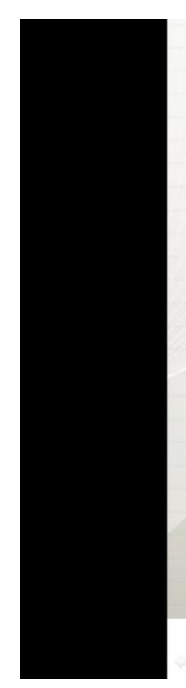

\section{My best friend and I}

- My name N... and ten yeres old my hers fair and aus braun my brather name Nick my best friend name Maria and ten yeres old. She like play volley boll hers raed and aus braun

\section{ᄀ.}

Eıкóva 5

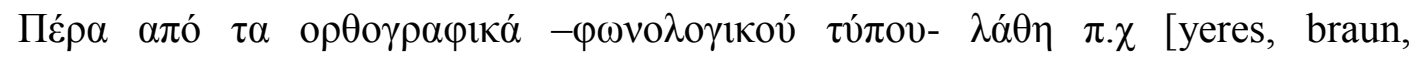

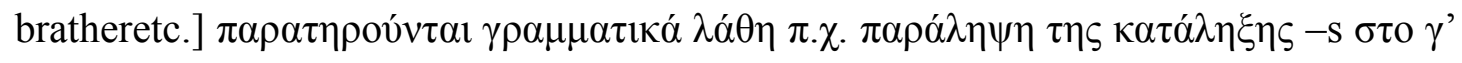

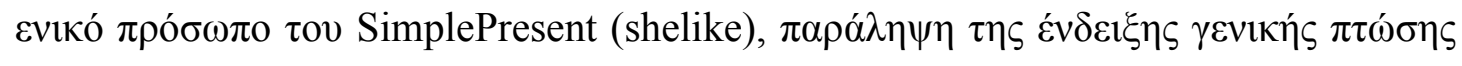

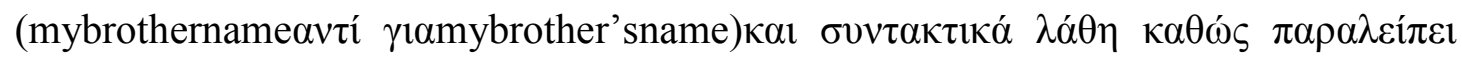

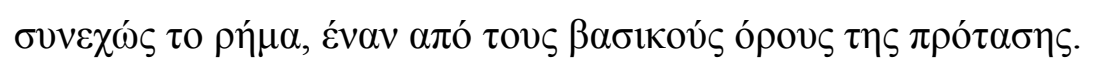




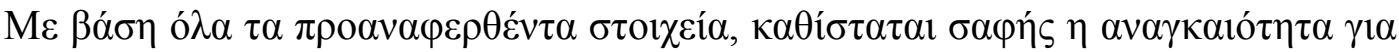

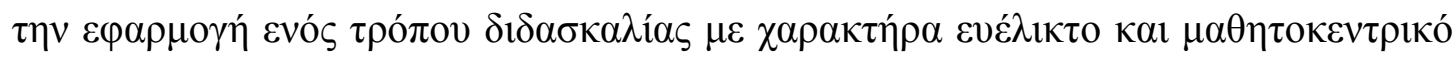

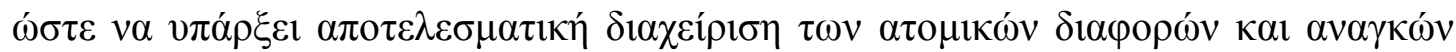

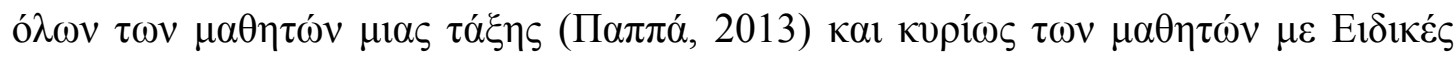

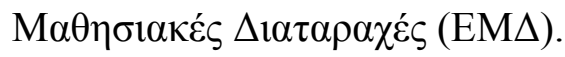

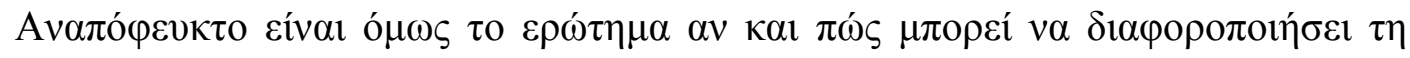

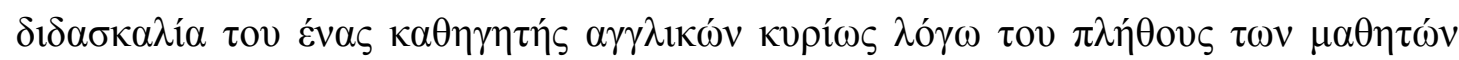

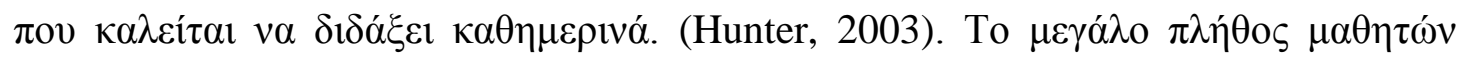

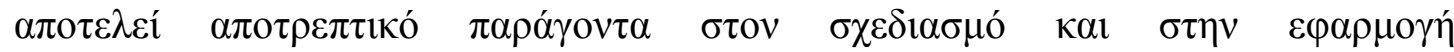

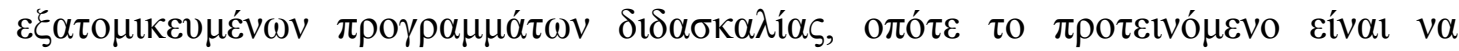

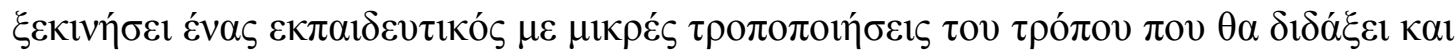

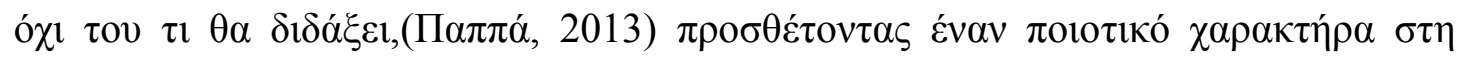

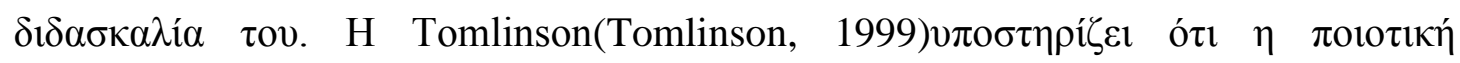

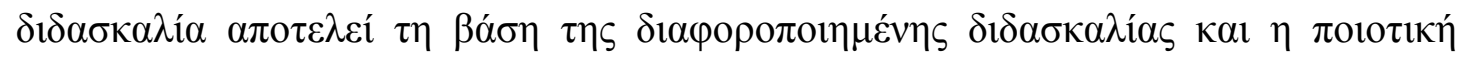

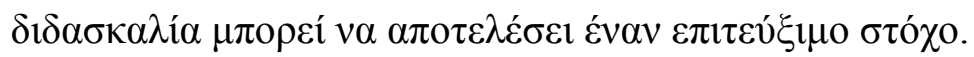

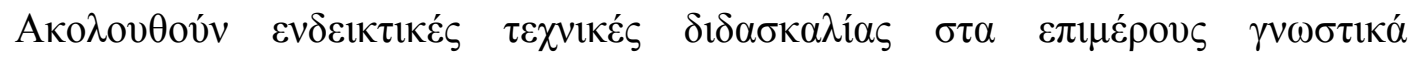

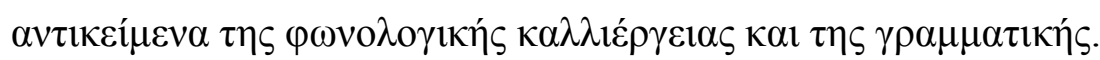

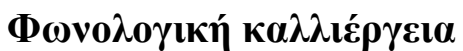

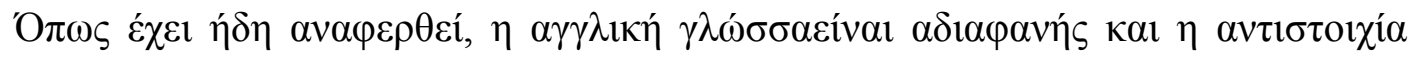

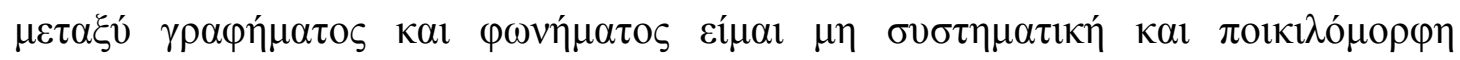

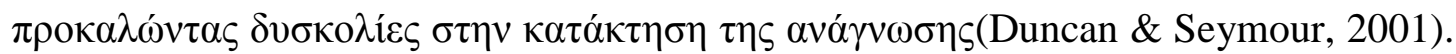

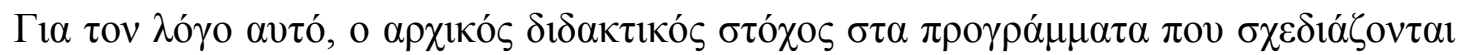

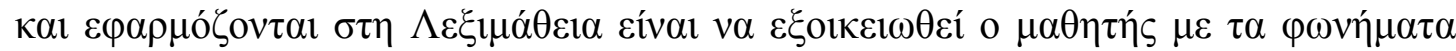

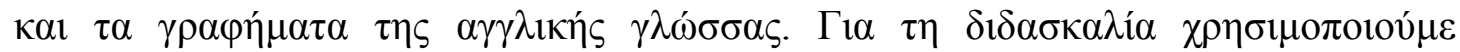

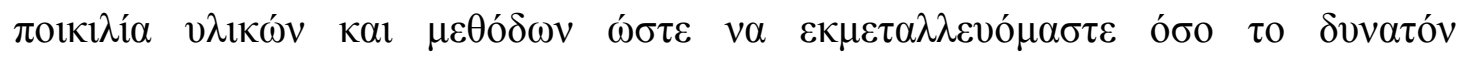

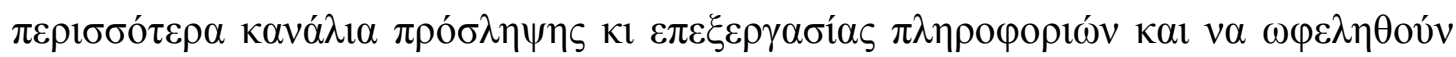

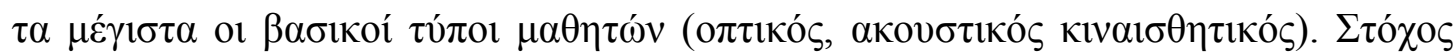

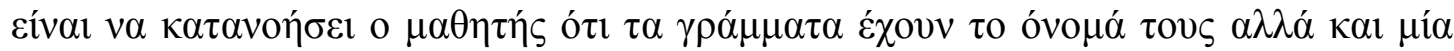

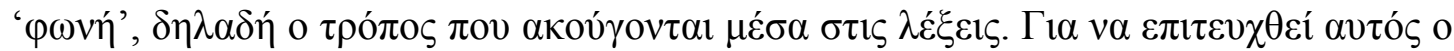

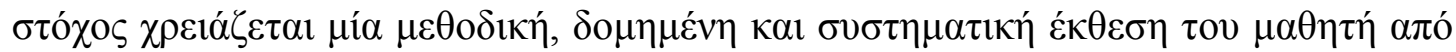




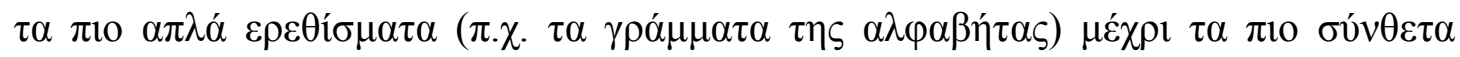

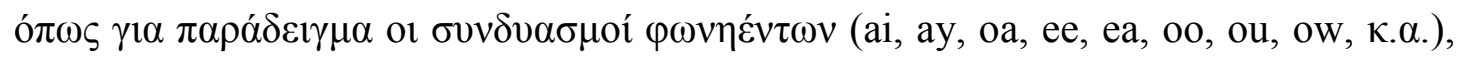

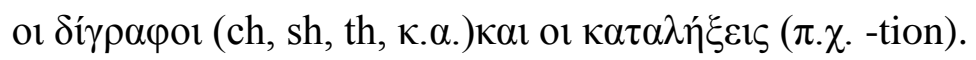

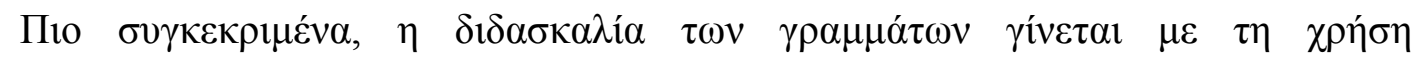

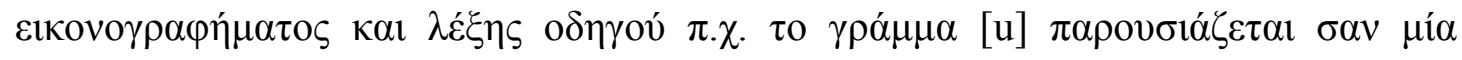

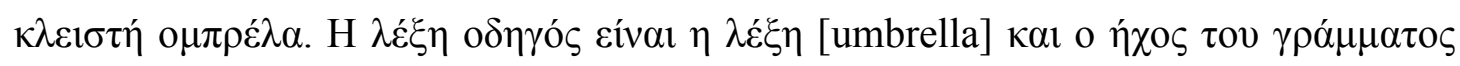

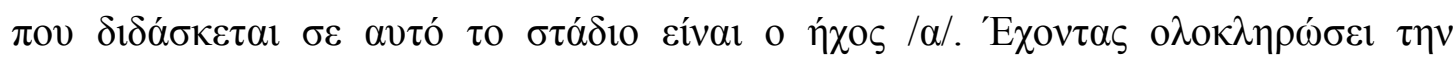

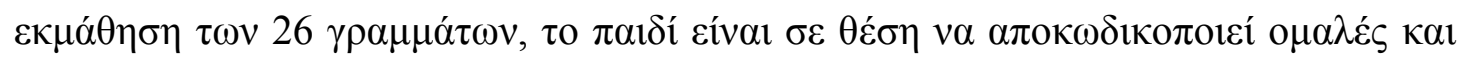

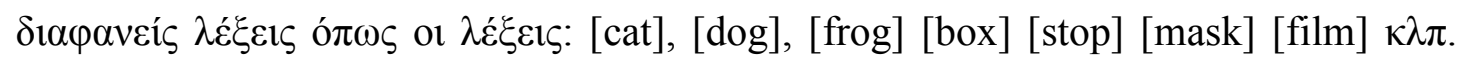

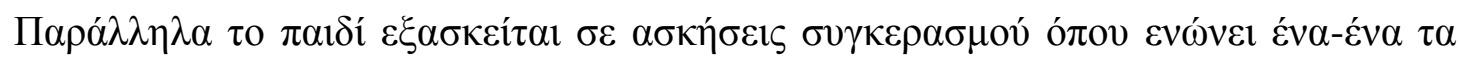

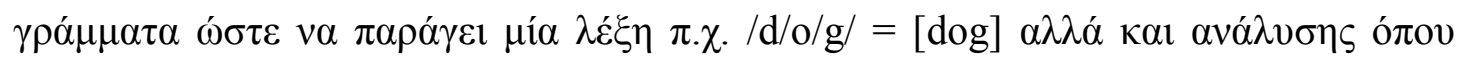

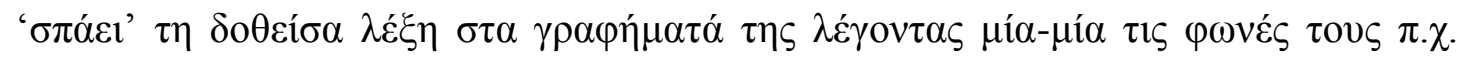
$[$ frog $]=$ f-r-o-g

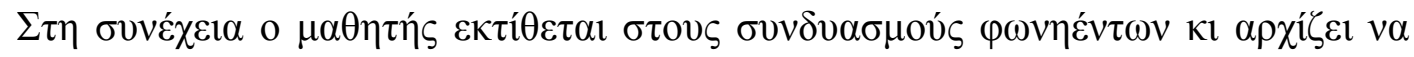

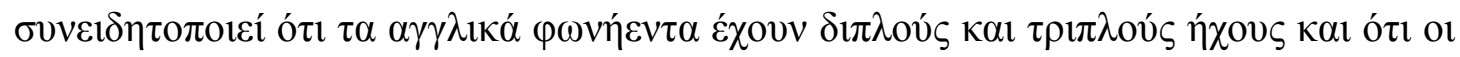

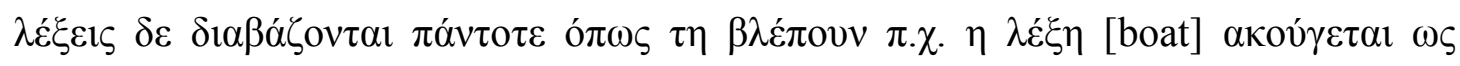

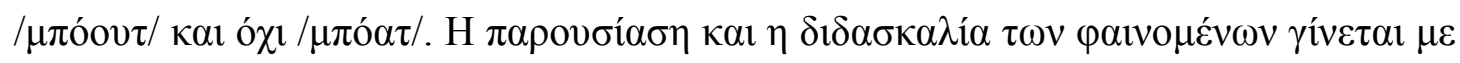

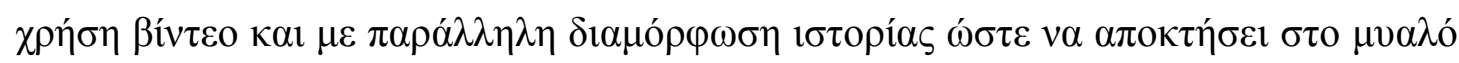

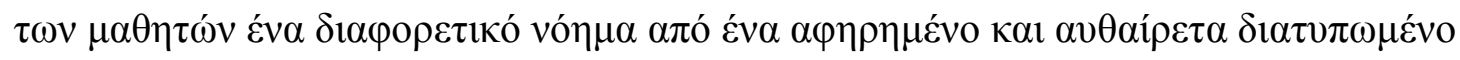

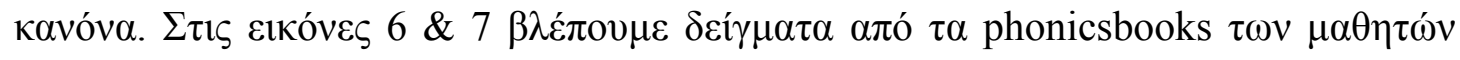

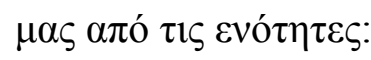

- Two vowels go walking (ea, ee, oa, ue, ) દ1кóva6

- The magic -e عıкóva 7

- Vowel combination-ou -oweı́óva 7

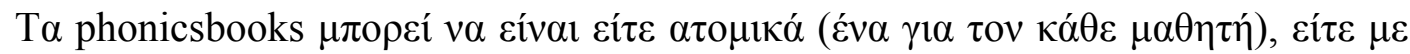

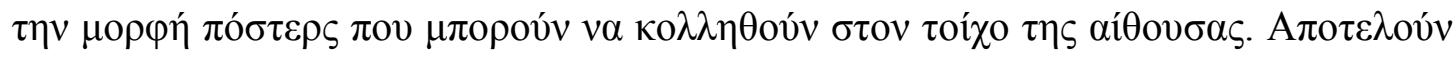

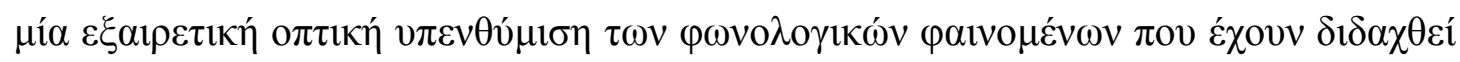

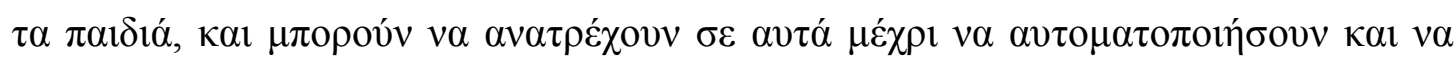

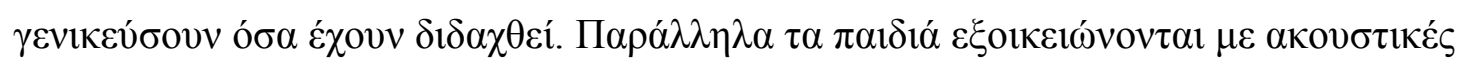

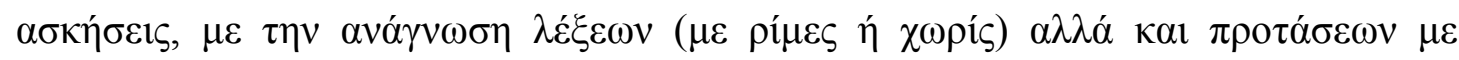

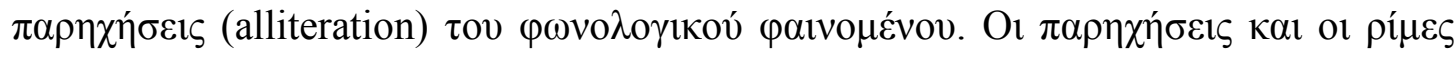




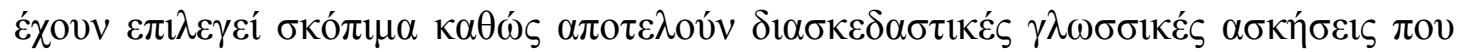

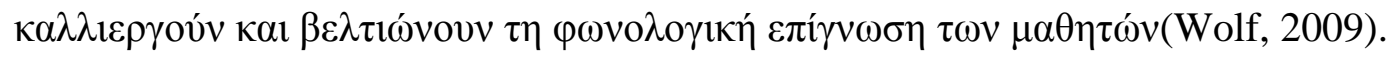

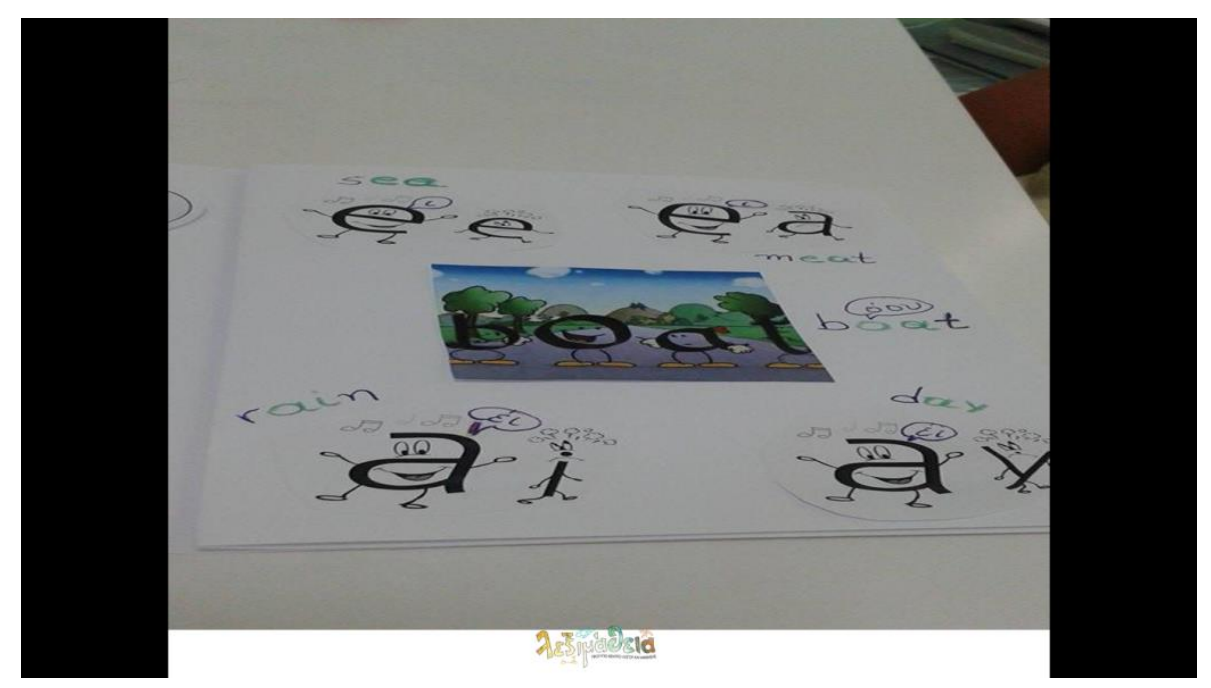

Eıкóva 6

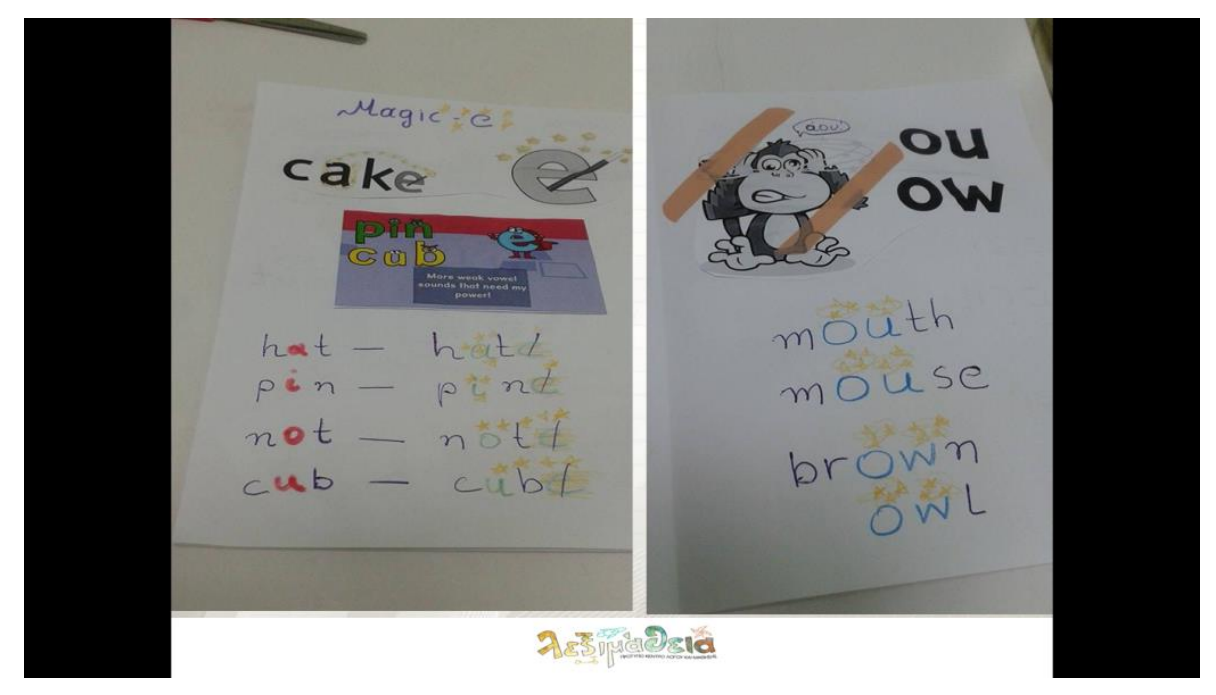

Eıкóva 7

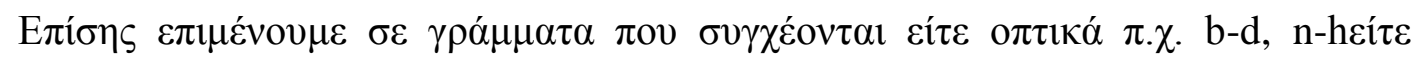

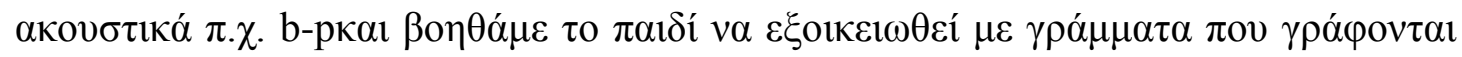

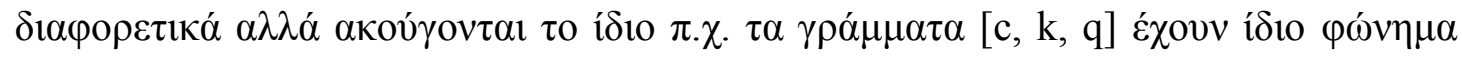
$\alpha \lambda \lambda \alpha \dot{\alpha} \delta 1 \alpha \varphi о \rho \varepsilon \tau 1 \kappa \alpha ́ ~ \gamma \rho \alpha \varphi \eta ́ \mu \alpha \tau \alpha$. 


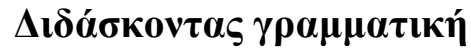

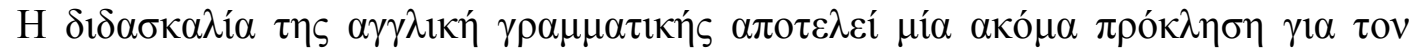

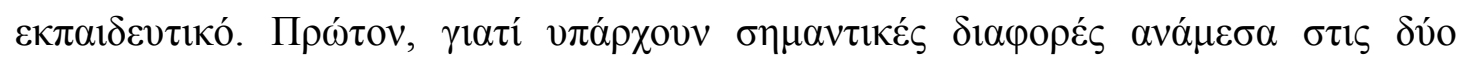

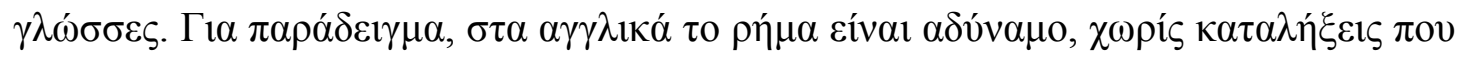

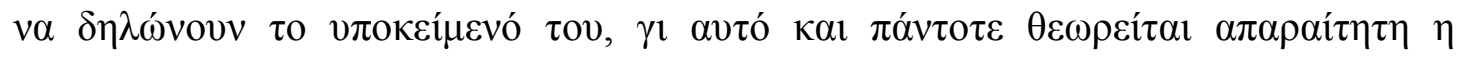

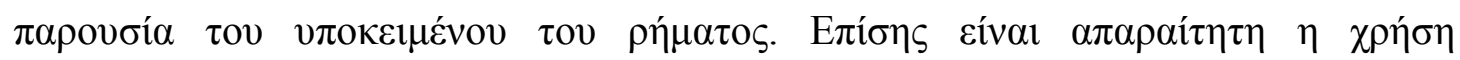

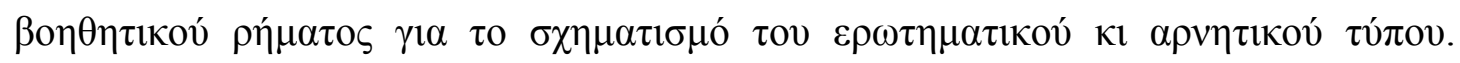

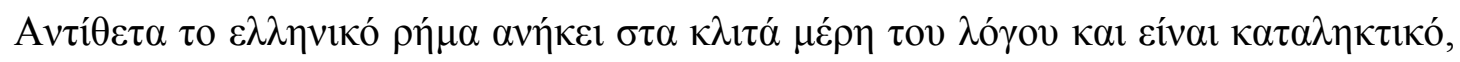

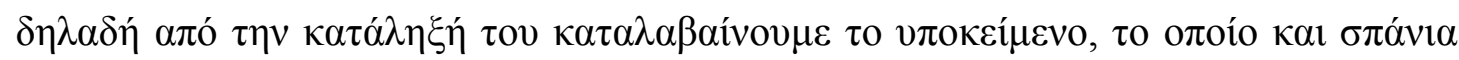

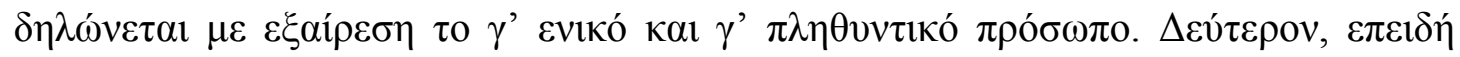

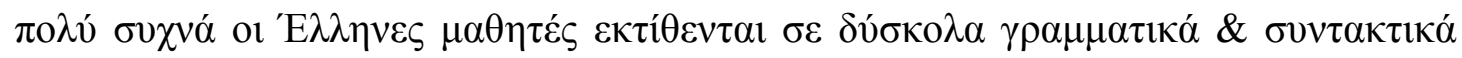

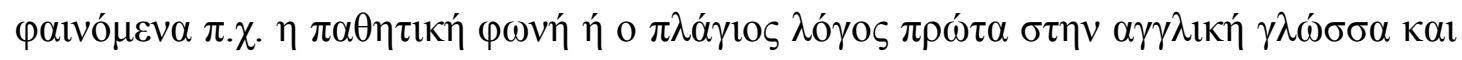

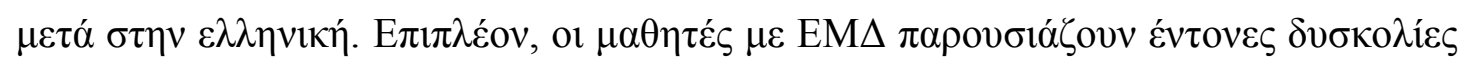

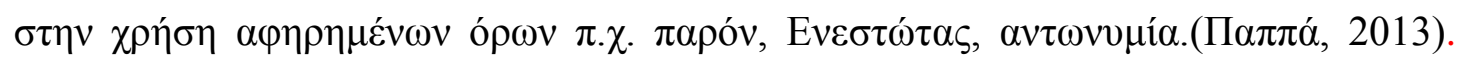

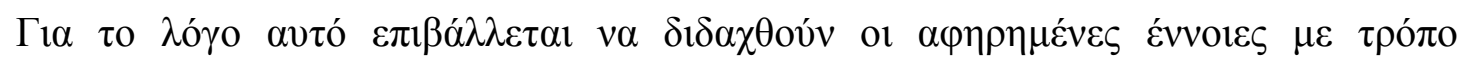

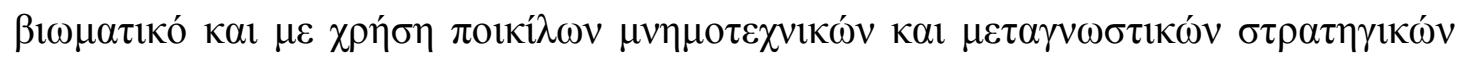

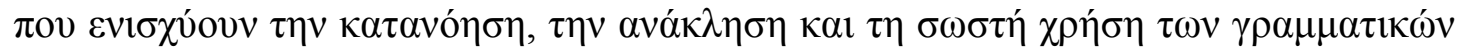

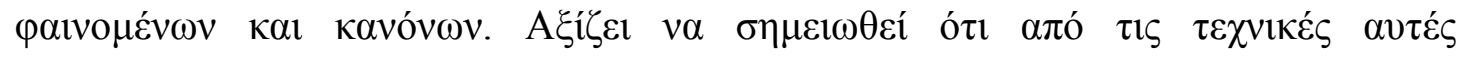

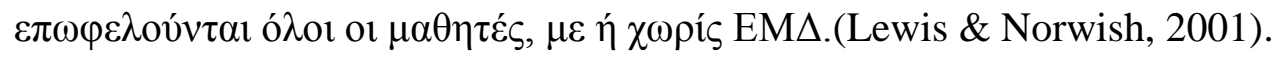

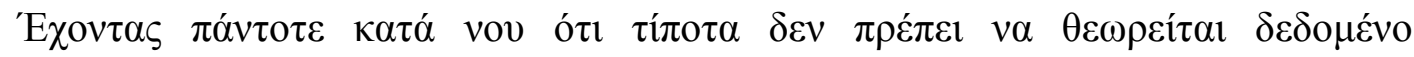

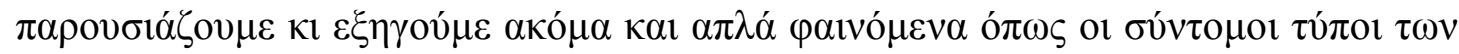

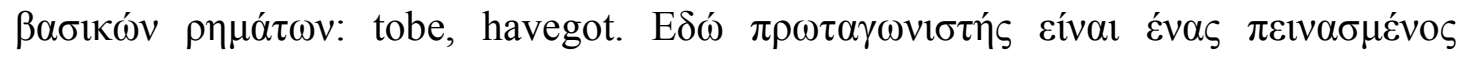

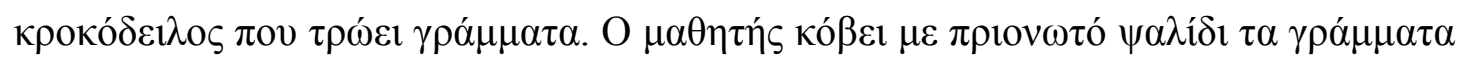

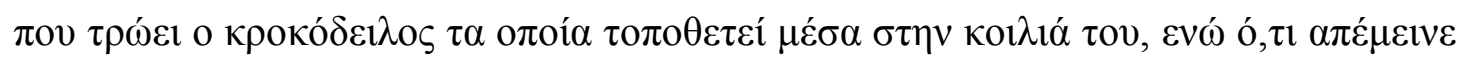

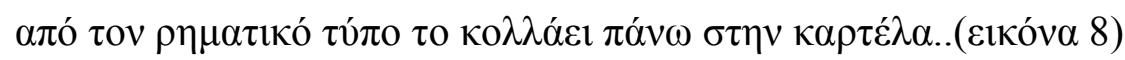




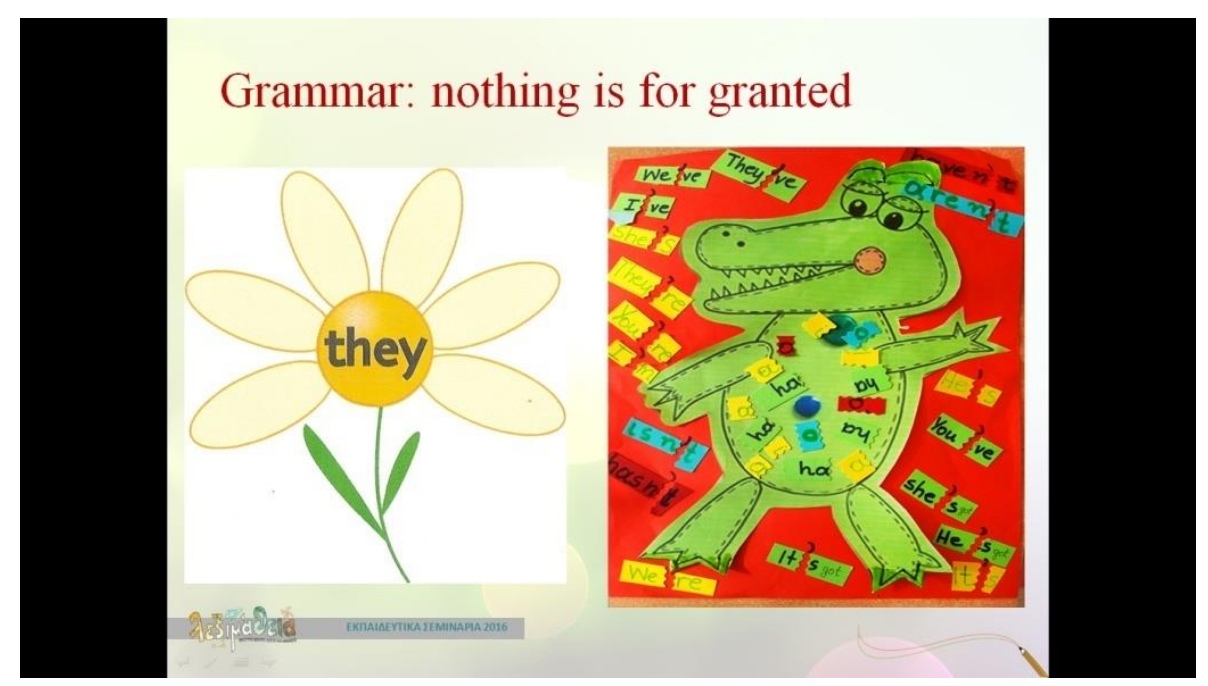

Eıкóva 8

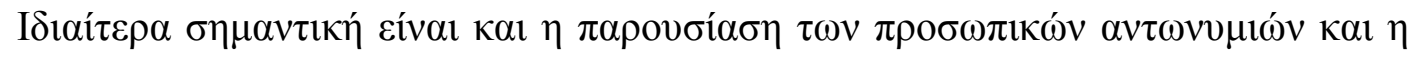

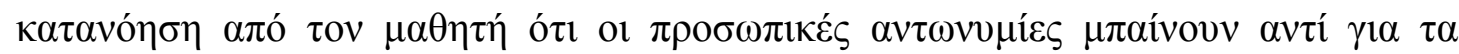

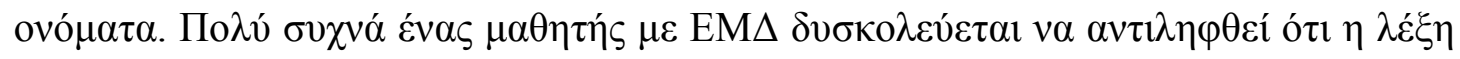

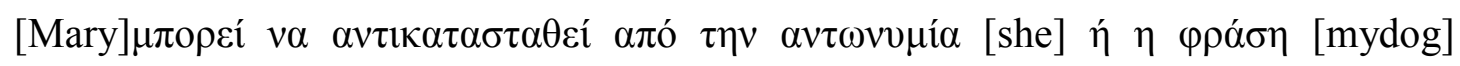
$\mu \pi$

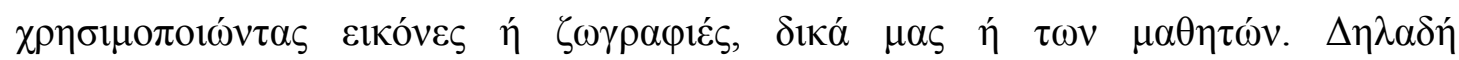

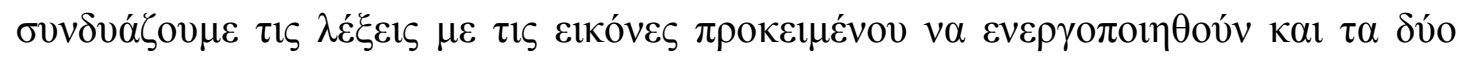

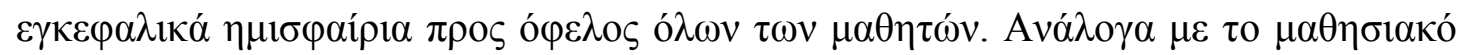

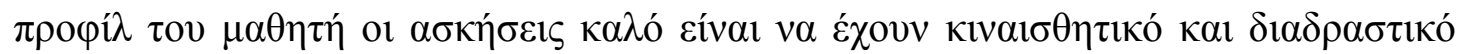

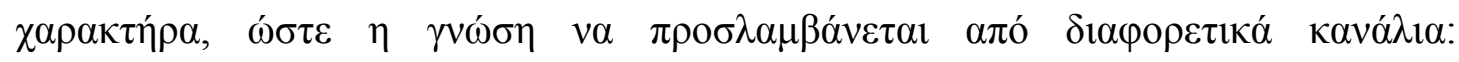

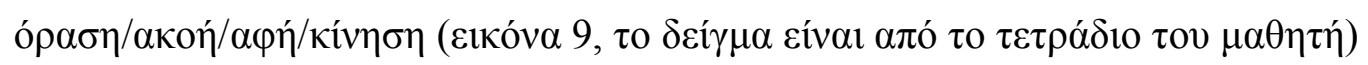




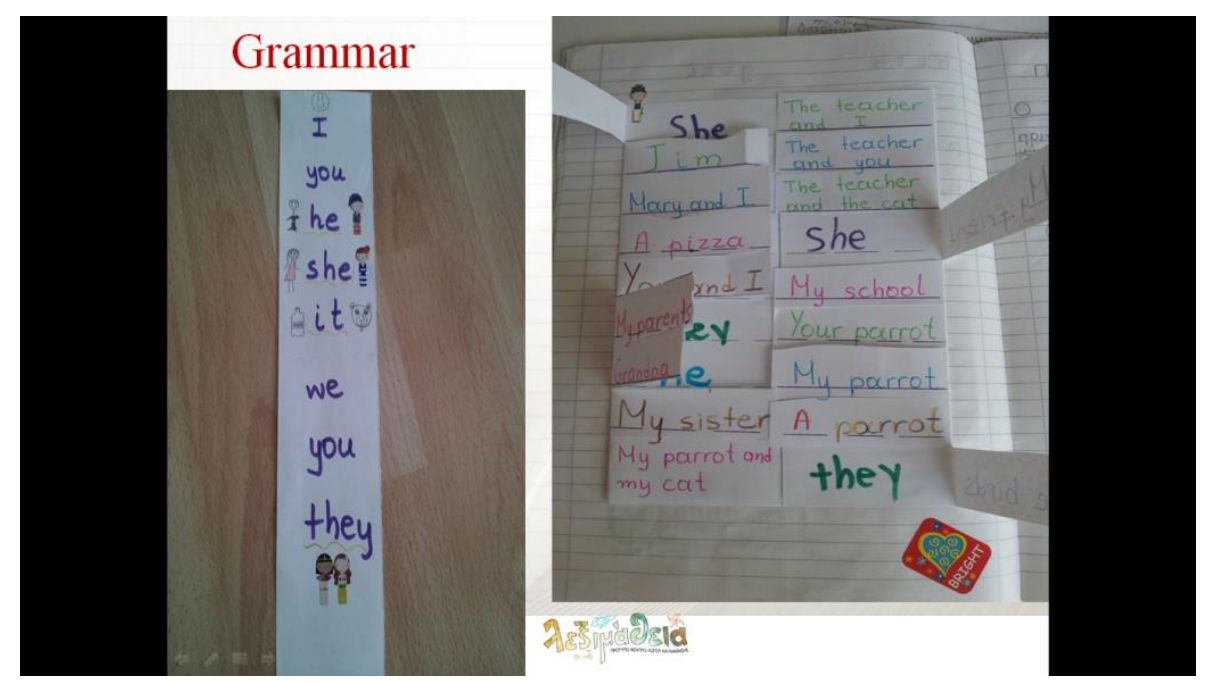

Eıкóva 9

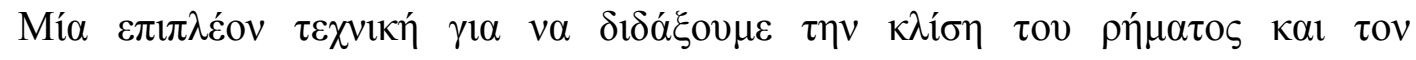

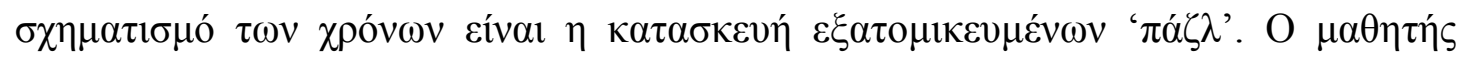

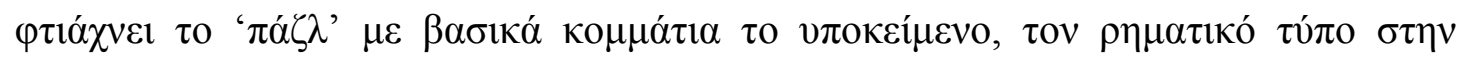

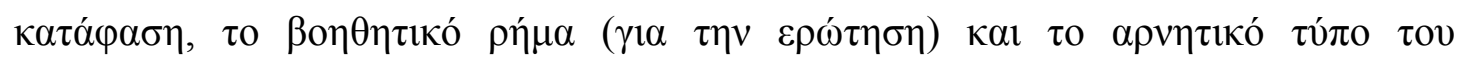

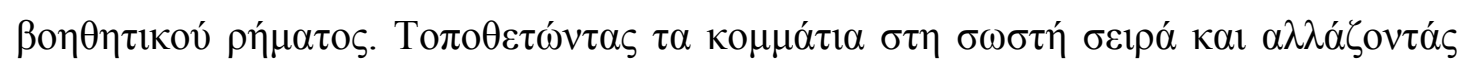

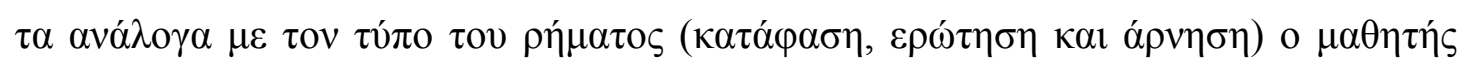

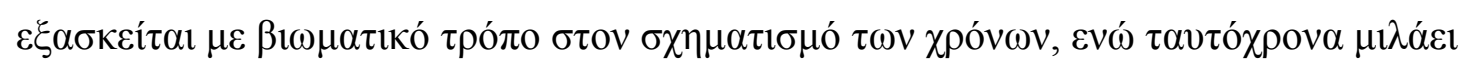

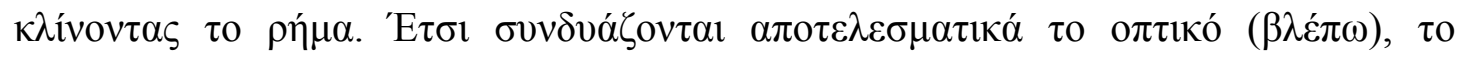

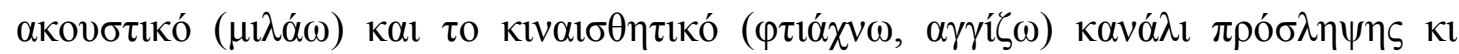

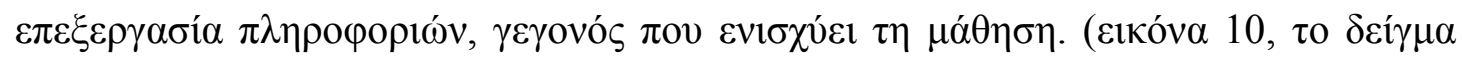

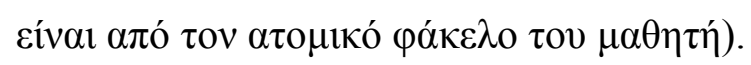




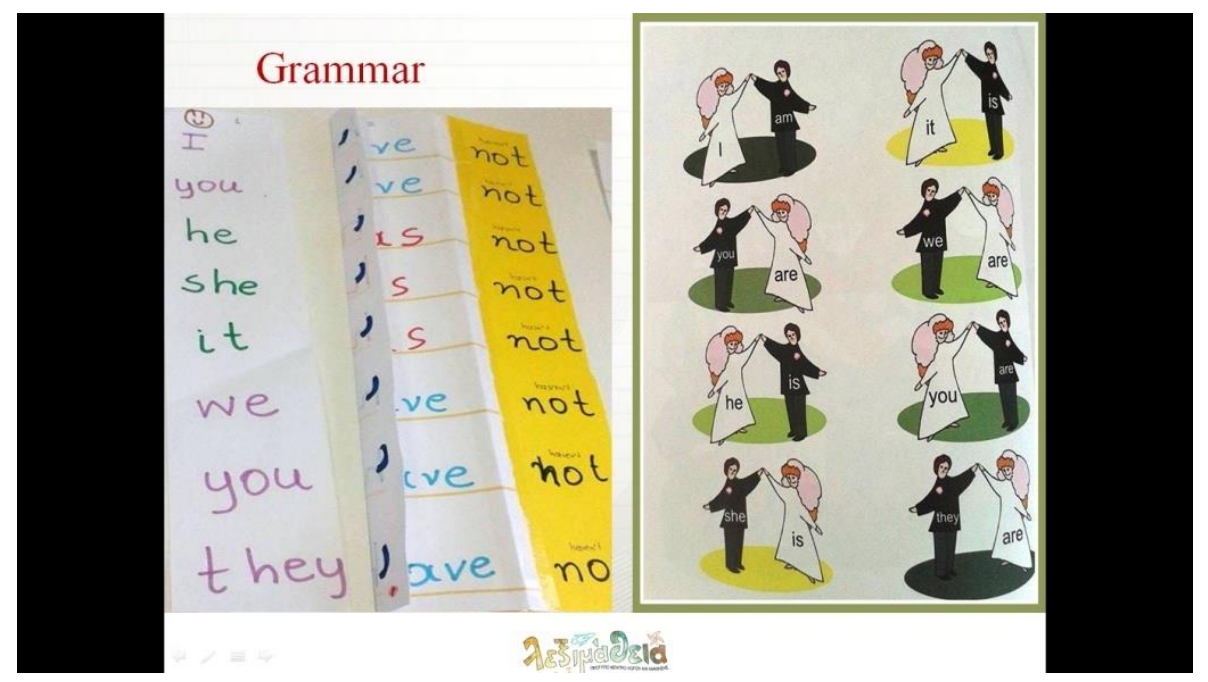

Eıкóva 10

\section{Eлí́loyos}

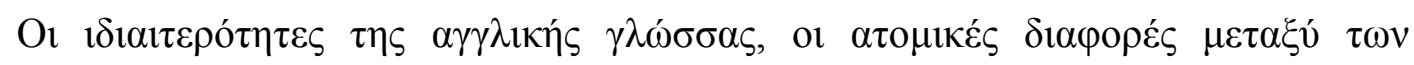

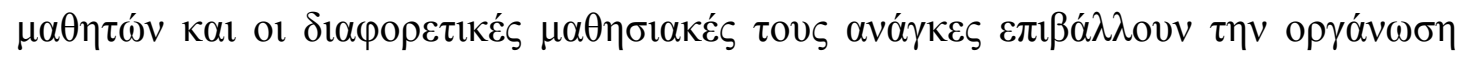

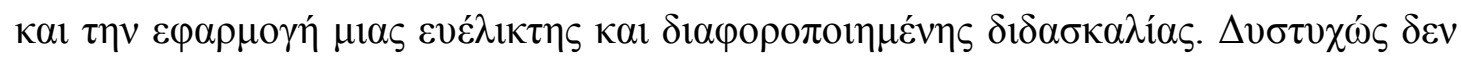

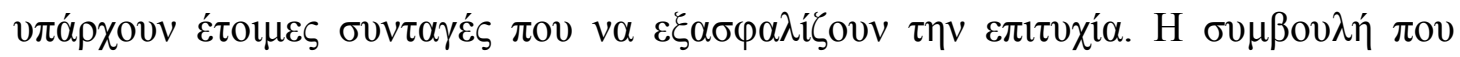

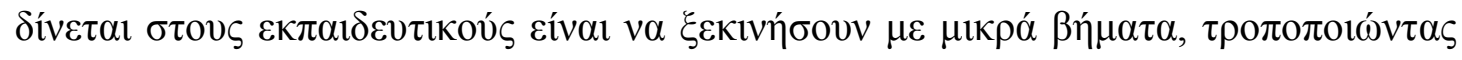

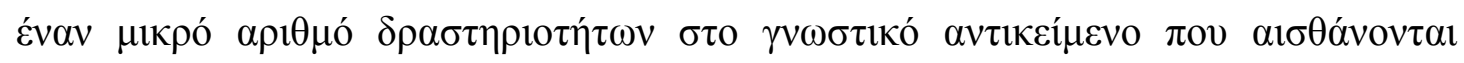

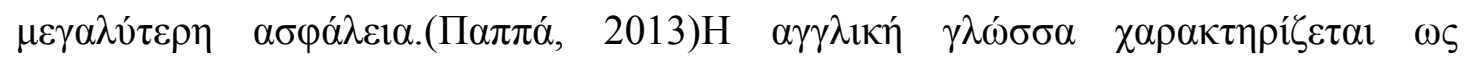

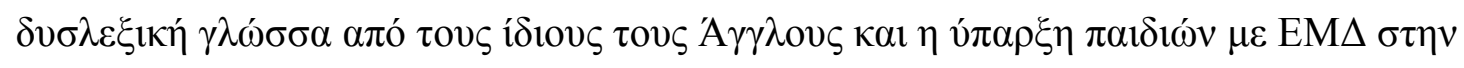

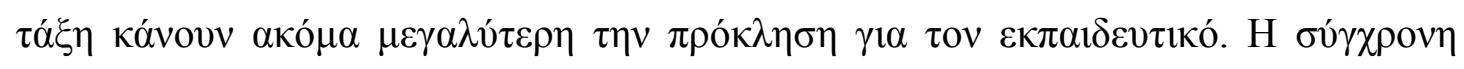

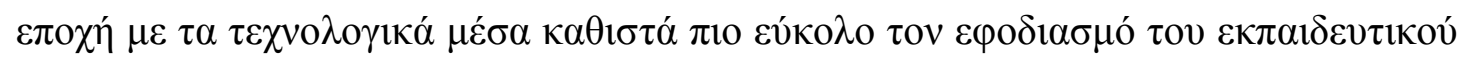

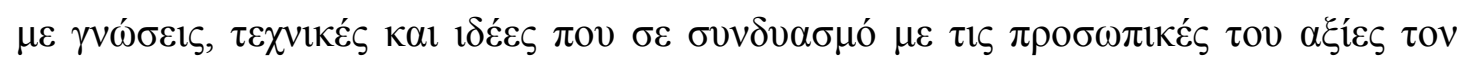

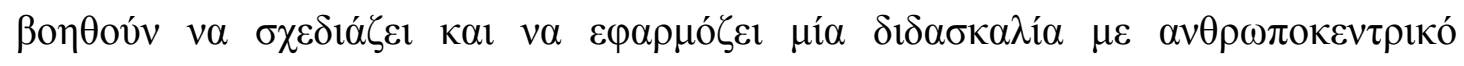

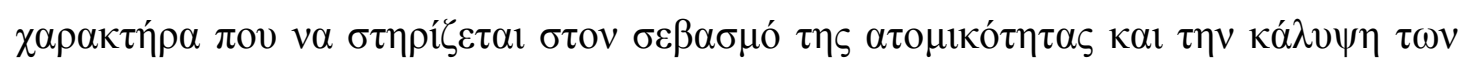

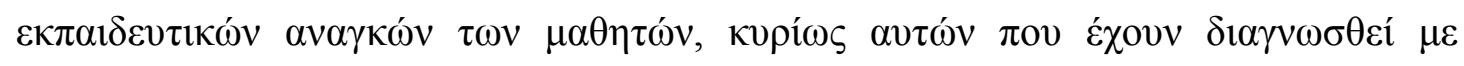

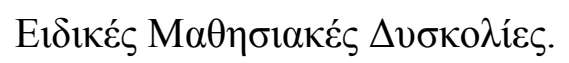




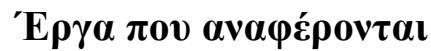

Chomsky, C. (1972, April). Stages in language development and reading exposure. Harvard Educational Review , oб. 1-33.

Chomsky, N. (1968). The sound pattern of English. New York: Harper \& Row.

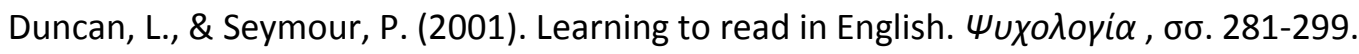

Frith, U. (1985). Beneath the surface of developmental dyslexia. ¿to J. \&. K. Patterson, Surfase dyslexia, neuropsychological and cognitive studies of phonological reading ( $\sigma \sigma .301-$ 330). London: Erlbaum.

Hunter, B. K.-S. (2003). Differentiated instruction in the English classroom. Content, process, product and assessment. Portsmouth: $\mathrm{NH}$ : Heinemann.

Lewis, A., \& Norwish, B. (2001). A critical review of systematic evidence concerning didtinctive pedagogies for pupils with difficulties in learning. Journal of researc on special education , $\sigma \sigma .313-329$.

Raderson, J. (2001, September 04). Newscientist life. Avóktnonaró https://www.newscientist.com/: https://www.newscientist.com/article/dn1233-english-istoughest-european-language-to-read/

Tomlinson, C. A. (1999). The differentiated classroom: Responded to the needs of all learners.USA: ASCD.

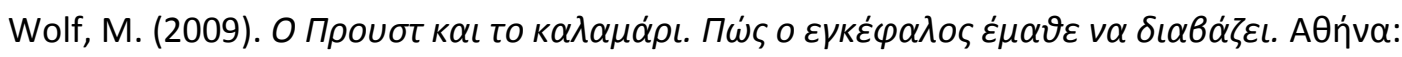
Пато́кп५.

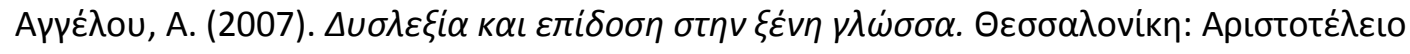

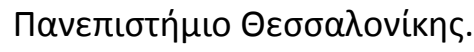




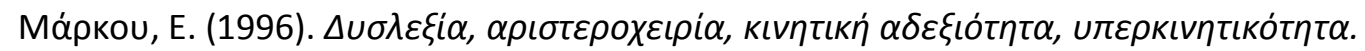

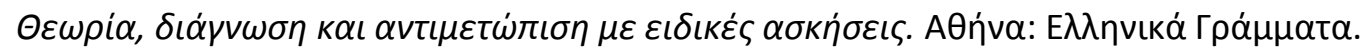

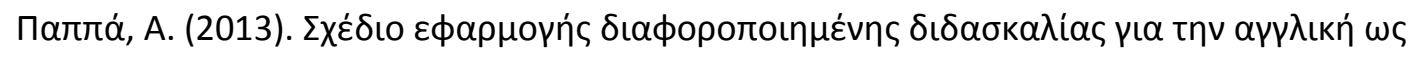

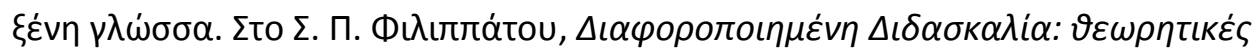

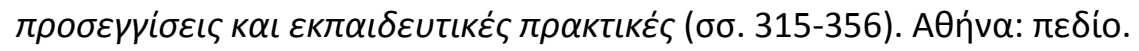

